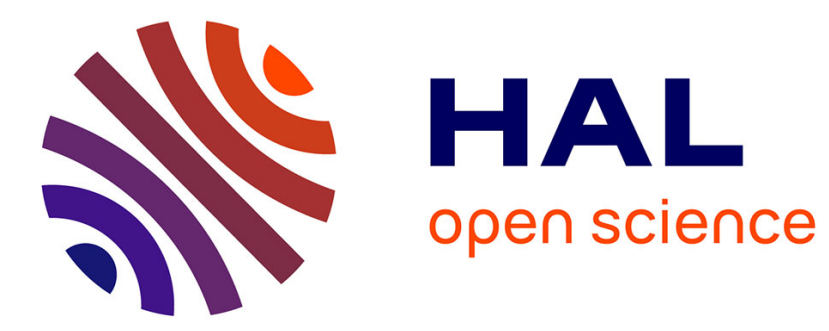

\title{
Return flux budget of polychromatic laser guide stars
}

\author{
Hugues Guillet de Chatellus, Jean-Paul Pique, Ioana Cristina Moldovan
}

\section{To cite this version:}

Hugues Guillet de Chatellus, Jean-Paul Pique, Ioana Cristina Moldovan. Return flux budget of polychromatic laser guide stars. Journal of the Optical Society of America, 2008, 25 (2), pp.400-415. 10.1364/JOSAA.25.000400 . hal-01118444

\section{HAL Id: hal-01118444 \\ https://hal.science/hal-01118444}

Submitted on 3 Mar 2015

HAL is a multi-disciplinary open access archive for the deposit and dissemination of scientific research documents, whether they are published or not. The documents may come from teaching and research institutions in France or abroad, or from public or private research centers.
L'archive ouverte pluridisciplinaire HAL, est destinée au dépôt et à la diffusion de documents scientifiques de niveau recherche, publiés ou non, émanant des établissements d'enseignement et de recherche français ou étrangers, des laboratoires publics ou privés. 


\title{
Return flux budget of polychromatic laser guide stars
}

\author{
Hugues Guillet de Chatellus*, Jean-Paul Pique and Ioana Cristina Moldovan \\ Laboratoire de Spectrométrie Physique, UMR 5588, CNRS-Université Joseph Fourier
}

140 avenue de la Physique, BP 87 -38402 Saint Martin d'Hères, France

\begin{abstract}
Polychromatic laser guide star (PLGS) is one of the solutions proposed to extend the sky coverage by large telescopes to $100 \%$, by enabling a complete knowledge of all perturbation orders of the wavefront. The knowledge of the tip-tilt is deduced from the monitoring of the chromatic components of the PLGS, from $330 \mathrm{~nm}$ to the visible or near infrared. Here we study the original scheme to create the PLGS by resonant excitation of the mesospheric sodium by two pulsed lasers (tens of $\mathrm{kHz}$ rep. rate, tens of $\mathrm{W}$ average power, tens of ns pulse duration), at 589 and $569 \mathrm{~nm}$ respectively. The efficiency of this process is investigated numerically by means of both Bloch equations and rate equations models. The influence of numerous laser parameters is studied. In the best case, having optimized all laser parameters, the return flux at $330 \mathrm{~nm}$ should not exceed $710^{4}$ photons $/ \mathrm{s} / \mathrm{m}^{2}$ for $2 * 18 \mathrm{~W}$ laser average power at the mesosphere. This maximum is obtained for a modeless laser whose spot diameter corresponds to 4 times the diffraction limit. For a diffraction-limited spot, the return flux falls down to 4 $10^{4}$ photons $/ \mathrm{s} / \mathrm{m}^{2}$. (C) 2007 Optical Society of America
\end{abstract}

OCIS codes: 010.1080, 010.1330, 020.4180, 030.4070, 120.4640, 140.2050, 190.4180, 260.2510, 300.2530.

Adaptive optics $(\mathrm{AO})$ techniques require the presence of an intense light source in the vicinity of the object observed by the telescope [1]. This is a major limitation to complete sky coverage since bright stars are rare, especially at the galactic poles. Laser guide stars (LGS) enable to extend the sky coverage by providing an intense and positional light source [2]. However they cannot compensate for the so-called "tip-tilt" resulting in an uncertainty on the position of the laser beacon relative to the object [3]. On the other hand if one is able to create a polychromatic laser beacon, the absolute tip-tilt can be deduced from the chromatic tip-tilt [4]. The latter could be measured by pointing the relative displacement of the chromatic components of the PLGS on the camera. This technique is very attractive since it enables theoretically $100 \%$ sky coverage, provided the vibrations of the telescope responsible for an additional global displacement of the image can be measured or compensated. The key point of the feasibility is given by the precision requested on the measurement of the differential tip-tilt. The latter is directly linked to the return flux available, i.e. to the intensity of the polychromatic laser guide star, and to the size of the laser spot in the sodium layer. The French project ELPOA (for Polychromatic Laser Guide Star for Adaptive Optics) is originally based on the two photon excitation of the mesospheric sodium, by means of two lasers, respectively at 589 and $569 \mathrm{~nm}$ [5]. Recently, another excitation scheme has been proposed: the direct excitation of the mesospheric sodium at $330 \mathrm{~nm}$. A detailed study of this latter process has been published a short while ago [6]. In the present paper, we focus on the two-photon excitation scheme, which has been partially investigated [7], but for which no extensive study has been published yet. We choose to restrict ourselves to dye amplified lasers with pulse duration of tens of $\mathrm{ns}$ and a few tens of $\mathrm{kHz}$ repetition rate.

The paper is organized as follows. First we recall some of the properties of this double excitation and we present the two 
numerical models that are used to calculate the efficiency of the process. The first one is the Beacon code, based on the resolution of Bloch equations, and developed by the CEA ("Commissariat à l'Energie Atomique") [8]. We slightly modified the code by adding an additional routine to take into account a multimode laser and a modeless laser we specifically developed for the ELPOA project. The second one is a rate equations model developed in our research group [6]. Both models are validated by comparing identical situations.

In the second part, we develop some straightforward aspects of the return flux by calculating asymptotic and "best case" expectations of the return flux, and we define the relations between the experimental parameters of the excitation. In the third part, we focus on the choice of the best laser configuration (single-mode, phase modulated, modeless and multimode). In the last part, we optimize the return flux by investigating the influence of different laser parameters (polarization, spectral width, pulse duration, delay and repartition of the energy between the two lasers). We finally give a maximum value of the return flux for a given (reasonable) laser power.

\section{Introduction}

\subsection{Two photon resonant excitation of the mesospheric sodium}

\subsubsection{Generalities}

The mesospheric sodium layer has been a subject of intense studies for several years [9]. It is now generally agreed that it is located on average at $90 \mathrm{~km}$ above the sea level with a height of $5-10 \mathrm{~km}$. The position of the layer varies continuously and presents intriguing dynamic phenomena: the so-called sodium sporadics [10]. Its column concentration varies with the seasons, between 2 and $8.10^{9}$ atoms $/ \mathrm{cm}^{2}$. In the remaining of the paper, we take the value of $4.10^{9}$ atoms $/ \mathrm{cm}^{2}$.

The original idea of polychromatic laser guide stars is based on the excitation of the $4 \mathrm{D}_{5 / 2}$ level of the sodium atom by a double resonant excitation of the $3 \mathrm{~S}_{1 / 2}-3 \mathrm{P}_{3 / 2}$ transition at $589 \mathrm{~nm}\left(\mathrm{D}_{2}\right.$ line) followed by the $3 \mathrm{P}_{3 / 2}-4 \mathrm{D}_{5 / 2}$ transition at $569 \mathrm{~nm}$ (actually $568.8 \mathrm{~nm}$ ) [4]. Other two-photon excitation schemes have been proposed, such as the non-resonant absorption of two photons at $578 \mathrm{~nm}$ but in general they require much higher peak powers and lasers in the picosecond regime with low repetition rate [11]. We chose not to discuss this case in this paper and we focus on the resonant case. The atom in the $4 \mathrm{D}_{5 / 2}$ level decays according to a radiative cascade providing lines at $330.3 \mathrm{~nm}$, $568.8 \mathrm{~nm}, 589.0 \mathrm{~nm}, 589.6 \mathrm{~nm}, 1138 \mathrm{~nm}, 1140 \mathrm{~nm}, 2208 \mathrm{~nm}$ and $2338 \mathrm{~nm}$. To measure a significant variation of the differential (chromatic) tilt, one need to consider the $330 \mathrm{~nm}$ line, and a lower frequency line $(589.6 \mathrm{~nm}$ or $2338 \mathrm{~nm}$ ) (see fig. 1). Therefore in the remaining of the paper, the flux budget for the polychromatic LGS will be characterized by the return flux at $330 \mathrm{~nm}$. We also present the results for the return flux at $589 \mathrm{~nm}$, since it might be of interest for the monochromatic LGS community: we consider that the irradiation by the second laser has a limited impact on the return flux at $589 \mathrm{~nm}$, and that the results presented in this paper on the return flux at $589 \mathrm{~nm}$ can also apply for monochromatic LGS. We also make the hypothesis that the transmission of the atmosphere on the return trip at $330 \mathrm{~nm}$ and the total efficiency of the detection apparatus are equal to $100 \%$. We neglect any non-radiative process. For simplicity reasons we also consider the situation where the laser power is constant over the shined area (i.e. "top-hat" spatial profiles are assumed for both lasers). This hypothesis is discussed in $\S 4.2$, where we compare the "top-hat" approximation with gaussian-shaped laser beams. All fluxes are given in number of photons per second and per square meter of reception telescope. They are deduced from the probability of emission by an individual sodium atom by:

$\Phi_{\lambda}=\frac{f . d_{N a} \cdot S}{D^{2}} p$

where :

- $\Phi_{\lambda}$ (in photons $\left./ \mathrm{s} / \mathrm{m}^{2}\right)$ is the return flux at the wavelength $\lambda$ (in $\mathrm{nm}$ ).

- $f$ is the laser repetition rate.

- $d_{N a}$ is the surface sodium density in the mesosphere $\left(d_{N a}=4.10^{13}\right.$ atoms $\left./ \mathrm{m}^{2}\right)$.

- $S$ is the size of the laser spot in the mesosphere $\left(\mathrm{m}^{2}\right)$.

- $D$ is the distance from the ground level to the mesosphere ( $\left.D=90.10^{3} \mathrm{~m}\right)$.

- $p$ is the probability of photonic emission per steradian, per atom and per laser pulse.
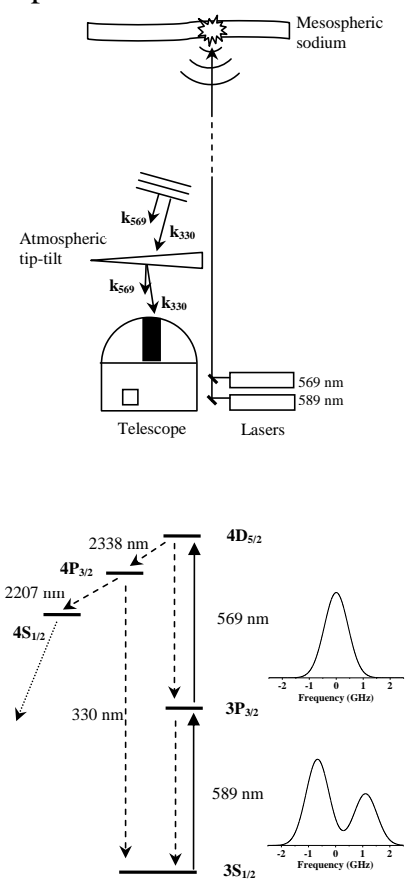

Fig. 1 Overview of the two-photon PLGS. The chromatic components of the PLGS are monitored by the telescope (top). The scheme is based on the twophoton resonant excitation of the mesospheric sodium (bottom). The Dopplerhyperfine widths of the transitions are given in GHz. 
1.1.2 Physical parameters of sodium transitions

The probability of excitation of the sodium atom is linked to the physical parameters of the transitions involved. The Beacon code considers the dipole moment whereas the rate equations model uses the absorption cross section. In this paragraph we recall the relation between these parameters for the transitions of the sodium atom and we give coherent numerical values to compare both models.

\subsubsection{Dipole moment}

The probability for an atom to undergo a spontaneous transition from an excited sublevel $m_{b}$ to a sublevel $m_{a}$ is according to [12]:

$$
A\left(m_{b} \rightarrow m_{a}\right)=\frac{k^{3}}{3 \pi \varepsilon_{0} \hbar}\left|\left\langle j_{b}, m_{b}\left|D_{q}\right| j_{a}, m_{a}\right\rangle\right|^{2}
$$

Using the Wigner-Eckart theorem, the Einstein coefficient $A_{b a}$ of the transition $b \rightarrow a$ is independent from $m_{b}$ and is given by:

$$
\begin{aligned}
A_{b a} & =\sum_{m_{a}} A\left(m_{b} \rightarrow m_{a}\right)=\frac{k^{3}}{3 \pi_{0} \hbar} \sum_{m_{a}, q}\left|\left\langle j_{a} 1 m_{a} q \mid j_{b} m_{b}\right\rangle\right|^{2} \\
& =\frac{k^{3}}{3 \pi \varepsilon_{0} \hbar} \frac{\left|\left\langle j_{b}\|D\| j_{a}\right\rangle\right|^{2}}{2 j_{b}+1}
\end{aligned}
$$

When level $b$ can decay to several $a_{i}$ lower levels (see fig. 2), the probability of the transition $b \rightarrow a_{i}$ is related to the radiative lifetime of level $b\left(\tau_{b}\right)$ by:

$$
A_{b a_{i}}=B r_{b a_{i}} \frac{1}{\tau_{b}}=B r_{b a_{i}} \Gamma_{b}^{\text {radiative }}
$$

where $B r_{b a_{i}}$ is the branching ratio from level $b$ to level $a_{i}$. The dipole moment can be expressed as:

$\left|\left\langle j_{b}\|D\| j_{a_{i}}\right\rangle\right|=\sqrt{\frac{3 \pi \lambda^{3} \varepsilon_{0} \hbar\left(2 j_{b}+1\right) B r_{b a_{i}}}{(2 \pi)^{3} \tau_{b}}}$

Note that a different convention sometimes makes use of the average dipole moment, defined as:

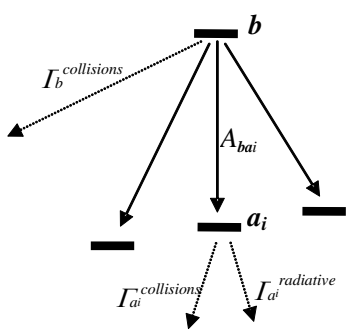

Fig. 2 Generic multilevel system. All states can decay through both radiative and non-radiative processes. $\overline{\mu_{b a_{i}}}=\frac{\left|\left\langle j_{b}\|D\| j_{a_{i}}\right\rangle\right|}{\sqrt{3\left(2 j_{a_{i}}+1\right)}}$

This is the case for instance in the Beacon program, where the average dipole moment expressed in Debye, is taken as a variable input.

\subsubsection{Expression of the absorption cross section}

\begin{tabular}{|l|l|l|}
\hline Transition $\mathrm{a}_{\mathrm{i}} \rightarrow \mathrm{b}$ & $3 \mathrm{~S}_{1 / 2} \rightarrow 3 \mathrm{P}_{3 / 2}$ & $3 \mathrm{P}_{3 / 2} \rightarrow 4 \mathrm{D}_{5 / 2}$ \\
\hline$\lambda$ & $589.0 \mathrm{~nm}$ & $568.8 \mathrm{~nm}$ \\
\hline $\mathrm{g}_{\mathrm{ai}}$ & 2 & 4 \\
\hline $\mathrm{g}_{\mathrm{b}}$ & 4 & 6 \\
\hline$\tau_{a_{i} \text { (ns) }}$ & $\infty$ & $\tau_{3 P_{3 / 2}=16 \mathrm{~ns}}$ \\
\hline$\tau_{b}$ (ns) & $\tau_{3 P_{3 / 2}=16 \mathrm{~ns}}$ & $\tau_{4 D_{5 / 2}}=54 \mathrm{~ns}$ \\
\hline Homogenous width (MHz) & $\Gamma_{1}=10 \mathrm{MHz}$ & $\Gamma_{2}=13 \mathrm{MHz}$ \\
\hline Mean dipole moment (Debye) & $\bar{\mu}_{1}=5.23 \mathrm{D}$ & $\bar{\mu}_{2}=1.91 \mathrm{D}$ \\
\hline Absorption cross section & $\sigma_{01}=1.1010^{-13} \mathrm{~m}^{2}$ & $\sigma_{02}=1.2110^{-14} \mathrm{~m}^{2}$ \\
& & \\
\hline
\end{tabular}

Table 1. Numerical values and writing conventions used in the following of the paper.

To link the dipole momentum to the differential absorption cross section, one needs to express the homogeneous linewidth of the $a_{i} \rightarrow b$ transition:

$\Gamma_{a_{i} b}=\Gamma_{a_{i}}^{\text {radiative }}+\Gamma_{b}^{\text {radiative }}+\Gamma^{\text {collisions }}+\ldots$

where $\Gamma_{a_{i}}^{\text {radiative }}=1 / \tau_{a_{i}}$ and $\Gamma_{b}^{\text {radiative }}=1 / \tau_{b}, \tau_{a_{i}}$ being the radiative lifetime of the level $a_{i}$ [13]. Since the collision rate in the mesosphere is much smaller than the homogeneous widths of the different transitions, the collisions can be neglected. For a lorentzian homogeneous line, the frequencyintegrated absorption cross section $\sigma_{0}=\int \sigma(\omega) d \omega$ is linked to the absorption cross section at the centre of the line $\sigma_{a_{i} b}$ by:

$\sigma_{a_{i} b}=\frac{2}{\pi \Gamma_{a_{i} b}} \sigma_{0}$

We also have [14]:

$$
\sigma_{0}=\frac{g_{b}}{4 g_{a_{i}}} \lambda^{2} A_{b a_{i}}
$$


where the $g$ coefficients represent the degeneracy of the levels; we obtain the relation:

$$
\sigma_{a_{i} b}=\frac{1}{2 \pi} \frac{g_{b}}{g_{a_{i}}} \lambda^{2} B r_{b a_{i}} \frac{\tau_{a_{i}}}{\tau_{a_{i}}+\tau_{b}}
$$

Numerical results are presented in table 1 .

\subsubsection{Laser parameters}

The particular set of wavelengths involved in the two-photon polychromatic laser guide star requires particular laser types and format. Up to now, the excitation of the mesospheric sodium at $589 \mathrm{~nm}$ to produce a LGS has been demonstrated both with dye, and solid state lasers. In the first case, laser physicists use the fact that the wavelength of the transition corresponds to the maximum of the fluorescence curve of rhodamine $6 \mathrm{G}$ pumped by a green laser (usually at $532 \mathrm{~nm}$ ) [15]. In the second case, two laser lines from a YAG crystal, respectively at $1.06 \mu \mathrm{m}$ and $1.32 \mu \mathrm{m}$ are mixed in a nonlinear crystal and provide $589 \mathrm{~nm}$ [16]. However for the second transition $(569 \mathrm{~nm})$ no such coincidence has been evidenced yet, excluding the use of simple solid state solutions for twophoton polychromatic laser guide stars. Dye lasers appear as the natural choice for the excitation at $569 \mathrm{~nm}$ for the same reasons as at $589 \mathrm{~nm}$. Concerning the optimization of the excitation of the sodium atom to the $4 \mathrm{D}_{5 / 2}$ level, the nonlinearity of the excitation requires high peak powers, limiting the use of $\mathrm{CW}$ lasers. For the same mean intensity, the gain in terms of peak power between a CW laser and a pulsed laser $(10 \mathrm{kHz}$ repetition rate, $50 \mathrm{~ns}$ pulse duration) is about 2000 , leading to a very large improvement $\left(>10^{6}\right)$ in terms of probability of two-photon absorption. Mode-locked ps lasers can also provide high peak powers but this possibility raises the difficulty of synchronising the $589 \mathrm{~nm}$ and $569 \mathrm{~nm}$ ps pulses with a precision better than a few ns, due to the fact that the radiative lifetime of the intermediate level is $16 \mathrm{~ns}$. Moreover, in order to overcome the saturation limit, a picosecond laser solution would require a very high repetition rate. On the other hand, pulsed dye lasers appear as the simplest and most natural choice, since there exist commercial solid state Q-switched pulsed pump lasers at $532 \mathrm{~nm}$, delivering more than $80 \mathrm{~W}$ average power. In the rest of the article, we restrict ourselves to dye systems, delivering a few tens of nanosecond pulses at a repetition rate in the range $10-30 \mathrm{kHz}$.

\subsection{Presentation of the Bloch equation model}

The Bloch equation model we consider is the Beacon code published in 2004 and available online [8]. It computes the population of all hyperfine sublevels of the $3 \mathrm{~S}_{1 / 2}, 3 \mathrm{P}_{3 / 2}, 4 \mathrm{D}_{5 / 2}$, $4 \mathrm{P}_{3 / 2}$ and $4 \mathrm{~S}_{1 / 2}$ levels. The laser atom interaction is treated in the semi-classical approximation (the atom is treated by the quantum formalism and the electromagnetic field is treated classically in the plane wave approximation). The Doppler broadening is also taken into account. The code considers numerous parameters, such as the laser power density (in $\mathrm{W} / \mathrm{cm}^{2}$ ), the temporal shape of the pulse (gaussian/ hypergaussian/square), the polarization, the spectrum (single mode or phase modulation), the pulse duration and the delay between the $589 \mathrm{~nm}$ and $569 \mathrm{~nm}$ laser pulses. The parameters of the electronic transitions (dipole moment, lifetimes, hyperfine coupling coefficients) are also taken as variables. The calculation of the emission probability of spontaneous photons takes into account the transitions between hyperfine levels according to generalized branching ratios. The angular distribution of photons is also considered. The output file returns the time evolution of the populations of the 48 hyperfine sublevels involved, the time probability of emission of photons at $589 \mathrm{~nm}, 569 \mathrm{~nm}$ and $330 \mathrm{~nm}$. It also recalls the real an imaginary parts of the laser fields, as well as their spectrum. Note that the Beacon code has been tested by its authors and successfully compared to another Bloch equations model developed by Morris, to account for the excitation of the monochromatic LGS at $589 \mathrm{~nm}$ [17].

\subsubsection{Limitations of the code}

\subsubsection{Hypotheses}

- Beacon does not take atomic collisions into account. Because of the low pressure in the mesosphere, collision processes occur on a time scale of hundreds of microsecond, much longer than any of the radiative processes involved in the fluorescence of the sodium atom.

- The Zeeman splitting of the hyperfine structure, due to the earth magnetic field, is also neglected.

- The level $4 \mathrm{D}_{3 / 2}$ is located less than $1 \mathrm{GHz}$ apart from the $4 \mathrm{D}_{5 / 2}$ level. But considering that the oscillator strength of the $3 \mathrm{P}_{3 / 2} \rightarrow 4 \mathrm{D}_{3 / 2}$ transition is ten times less than for the $3 \mathrm{P}_{3 / 2} \rightarrow 4 \mathrm{D}_{5 / 2}$ transition, this level has been neglected in the program.

- Finally the recoil experienced by the sodium atom is not taken into account. The energy shift associated is about $10 \mathrm{kHz}$ and is much smaller than the homogeneous widths of the atomic transitions considered. Since only pulsed excitations are considered, each atom experiences at best a few cycles of absorption/fluorescence per laser pulse, which excludes any cumulative effect.

\subsubsection{Restrictions}

- In order to limit the calculation time, the two-colour excitation process is treated distinctly from the radiative cascade. Namely the excitation phase is based on the numerical resolution of the Bloch equation for the 48 hyperfine sublevels of the energy levels $3 \mathrm{~S}_{1 / 2}, 3 \mathrm{P}_{3 / 2}$ and $4 \mathrm{D}_{5 / 2}$. Then the cascade is described by a rate equation model. During the first phase, the cascade (i.e. from the $4 \mathrm{D}_{5 / 2}$ level) is treated as a leaking. In the second step, this leaking is used to calculate the populations of the different levels involved in the cascade. This implies that there is no recycling of the populations involved in the leaking during the laser pulse. This brings a limitation to the duration of the laser pulses, which should not exceed a few times the radiative lifetime of the $4 \mathrm{D}_{5 / 2}$ level (i.e. $54 \mathrm{~ns}$ ). An additional restriction arises also for highly saturating laser pulses with a temporal gaussian shape. In that case, even if the duration of 
the laser pulse satisfies the previous limitation, the effective duration of the laser pulse seen from the sodium atom is increased because of the wings of the temporal Gaussian function. Therefore the results obtained by the Beacon code for strong laser pulses with a Gaussian shape have to be taken with caution. This does not apply to temporal square pulses.

- The radiative cascade $4 \mathrm{P}_{3 / 2} \rightarrow 3 \mathrm{D}_{3 / 2,5 / 2} \rightarrow 3 \mathrm{P}_{3 / 2,1 / 2} \rightarrow 3 \mathrm{~S}_{1 / 2}$ is neglected.

- Finally, the hyperfine structure of the $4 \mathrm{D}_{5 / 2}$ is taken into account, but all hyperfine levels are degenerate.

\subsubsection{Extrapolation for long times and validation}

The Beacon code is written in the Fortran language. Since the version available online is not parallelized, the average time of calculation (500 ns duration with $0.01 \mathrm{~ns}$ time step) is typically 24 hours for a $2.6 \mathrm{GHz}$ processor using full CPU resources. A longer time range would prohibit parametric studies. Therefore we chose to limit the time range to $500 \mathrm{~ns}$. The typical laser excitations considered here are shorter than 100 ns FWHM and we consider that after $300 \mathrm{~ns}$, the laser pulse has gone away. Then the atom relaxes according to the lifetimes of the levels involved in the cascade. We determine the asymptotic value of the probability of emission per atom after $500 \mathrm{~ns}$ by fitting the temporal probability of emission by exponential laws. Actually the difference between the probability emission at $500 \mathrm{~ns}$ and the asymptotic value never exceeds $7 \%$. Note also that this procedure concerns only the photons at $330 \mathrm{~nm}$, since the lifetime of the $4 \mathrm{P}_{3 / 2}$ level is $110 \mathrm{~ns}$. For the flux at $589 \mathrm{~nm}$ and $569 \mathrm{~nm}$, the emission probabilities have reached by far their asymptotic values at $500 \mathrm{~ns}$, since the lifetimes of the $3 \mathrm{P}_{3 / 2}$ and $4 \mathrm{D}_{5 / 2}$ are $16 \mathrm{~ns}$ and $54 \mathrm{~ns}$ respectively. Before starting intensive calculations, we performed several tests: we ran the Beacon code in the same conditions as in [8] and we obtained similar results. We also compared the values we obtained with some of the results given in [18] to account for the PLGS experiments (PASS-2) in 2000 in Pierrelatte, and we find an agreement better than $8 \%$.

\subsubsection{Modification of the Beacon code}

The Beacon code was developed initially to simulate the behaviour of the sodium atom when submitted to single mode or phase-modulated lasers. However, the development in our group of an intracavity FSF ("Frequency Shifted Feedback") or modeless- laser, lead us to include in the code an additional routine to simulate this particular light source [19]. The peculiarity of this laser is a continuous spectrum at the output, with no apparent mode structure. The modeless behaviour is obtained by inserting in the cavity an acousto-optics modulator operating at an RF frequency of $40 \mathrm{MHz}$. The function of this modulator is to increase the optical frequency of the photon each time it makes a roundtrip in the laser cavity. This prohibits destructive and constructive interferences: in other words, every spontaneous photon is supported and amplified by the cavity. To limit the spectral width, other selective elements are inserted in the laser cavity: a Lyot birefringent filter and a Fabry-Pérot etalon. For the laser at $589 \mathrm{~nm}$, the spectrum obtained has a $3 \mathrm{GHz}$ wide Gaussian-like profile. The $3 \mathrm{GHz}$ width is optimized to excite all the Doppler-hyperfine structure of the $3 \mathrm{~S}_{1 / 2} \rightarrow 3 \mathrm{P}_{3 / 2}$ transition. We also plan to develop a similar modeless laser at $569 \mathrm{~nm}$, with a $1 \mathrm{GHz}$ bandwidth (hyperfine width of the $3 \mathrm{P}_{3 / 2}$ and $4 \mathrm{D}_{5 / 2}$ levels + Doppler broadening).

The question is: how to include this particular modeless electromagnetic field in the Beacon code? Despite many studies published in the literature, there is no clear conclusion on the expression of the electric field at the output of the FSF laser. Some experiments lead to an incoherent spectrum [20], some others to a chirping comb [21]. We study two possible models for the electric field of the modeless laser.

\subsubsection{Narrow Free Spectral Range Model (NFSR)}

A way to describe a modeless laser is to write it as a multimode laser with a free spectral range (FSR) narrower than the homogenous line width of the atomic transition excited by the laser. In that case, we take into account the fact that the modeless laser can excite all velocity classes of sodium atoms. The homogeneous linewidth of the $3 \mathrm{~S}_{1 / 2} \rightarrow 3 \mathrm{P}_{3 / 2}\left(\right.$ resp. $3 \mathrm{P}_{3 / 2} \rightarrow$ $4 \mathrm{D}_{5 / 2}$ ) transition is $\Gamma_{1}=10 \mathrm{MHz}$ (resp. $\Gamma_{2}=13 \mathrm{MHz}$ ). We choose to model the modeless laser by a $2 \mathrm{MHz}$ free spectral range multimode laser. To avoid any artificial mode-locking artefact, we assume random phases between the modes. To check the consistency of our approach, we vary the spectral free range between $2 \mathrm{MHz}$ and $3 \mathrm{GHz}$. We observe that the return flux decreases when the free spectral range (FSR) exceeds a few tens of $\mathrm{MHz}$. For a $3 \mathrm{GHz}$ FSR, a single laser mode remains in the $3 \mathrm{GHz}$ wide $\mathrm{D}_{2}$ line and the same flux is obtained than for two single mode lasers.

\subsubsection{Chirping Comb Model (CCM)}

We compare the previous results with an alternative expression of the electric field of a FSF laser, consisting in a chirped comb spectrum. The expression we used is derived in [22]. The electric field is written as:

$$
E(t)=E_{0} \sum_{-\infty}^{+\infty} e^{-\left(t / t_{0}\right)^{2}} e^{-i \frac{\Delta}{2 \tau_{r}}\left(t-n \tau_{s}\right)^{2}} e^{i \omega_{\max }\left(t-n \tau_{s}\right)}
$$

with the following parameters:

$t_{0}=2 \pi \frac{\tau_{r}}{\Delta} \frac{\delta v}{\sqrt{2 \ln 2}}$

and

$\tau_{s}=2 \pi / \Delta$

- $\omega_{\max }$ is the central angular frequency of the laser.

- $\tau_{r}$ is the round trip time in the laser cavity (3.57 ns in our case).

- $\Delta$ is the shift in frequency experienced by a photon per round trip $(80 \mathrm{MHz})$.

- $\delta v$ is the laser spectral width of $3 \mathrm{GHz}$ for the $3 \mathrm{~S}_{1 / 2} \rightarrow 3 \mathrm{P}_{3 / 2}$ (resp. $1 \mathrm{GHz}$ for the $3 \mathrm{P}_{3 / 2} \rightarrow 4 \mathrm{D}_{5 / 2}$ transition). 
We compare the numerical results obtained using both models (fig. 3). We obtain a good agreement between them. In the following of the paper, we choose to consider the NFSR model to describe the electric field of the modeless laser.

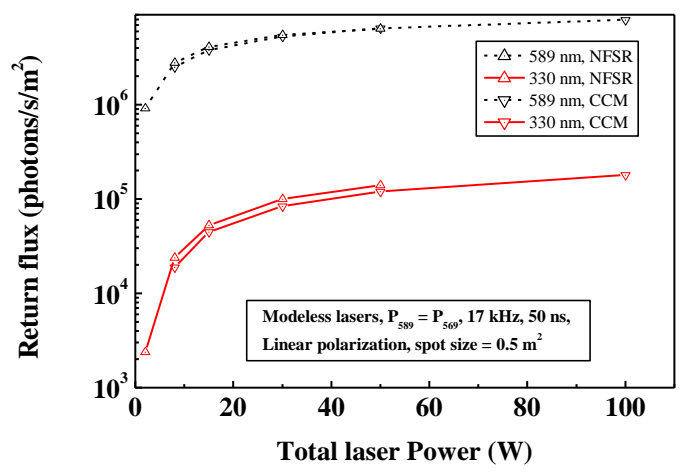

Fig. 3. Plot of the return fluorescence flux given by the Beacon code for both models of the modeless laser (Gaussian time profile, top-hat spatial profile).

1.3 Experimental validation and comparison with the rate equation model

\subsubsection{The rate equation model (REM)}

We proposed recently a different excitation scheme to create the PLGS, based on the direct excitation of the $4 \mathrm{P}_{3 / 2}$ level. We developed in [6] a REM model to compare both excitation schemes. Here we just recall the main definitions; additional details can be found in [6]. Fig. 4 shows the six levels simplification and notation.

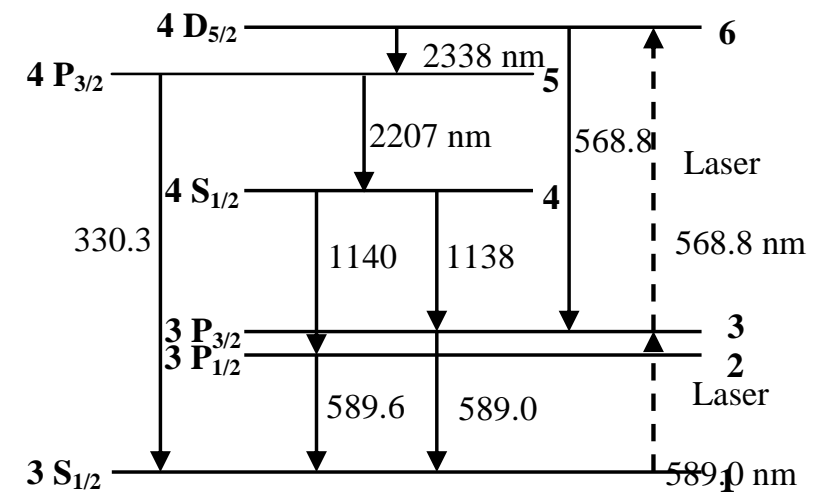

Fig. 4. Energy diagram and relaxation pathways of the two-photon excitation in the REM.

The six differential rate equations that describe the atomic system are:

$$
\begin{aligned}
& \frac{\partial N_{1}(t, r, v)}{d t}=-N_{1}(t, r, v) \int_{-\infty}^{+\infty} \sigma_{1}\left(v^{\prime}-v\right) \Phi_{1}^{L}\left(t, r, v^{\prime}\right) d v^{\prime} \quad \text { (absorption) } \\
& +\frac{N_{3}(t, r, v)}{\tau_{31}}+\frac{N_{2}(t, r, v)}{\tau_{21}}+\frac{N_{5}(t, r, v)}{\tau_{51}} \text { (spontaneous emission) }
\end{aligned}
$$

$$
\begin{aligned}
& +N_{3}(t, r, v) \frac{g_{1}}{g_{3}} \int_{-\infty}^{+\infty} \sigma_{1}\left(v^{\prime}-v\right) \Phi_{1}^{L}\left(t, r, v^{\prime}\right) d v^{\prime} \quad \text { (stimulated emission) } \\
& \frac{\partial N_{2}(t, r, v)}{d t}=\frac{N_{4}(t, r, v)}{\tau_{42}}-\frac{N_{2}(t, r, v)}{\tau_{21}} \\
& \frac{\partial N_{3}(t, r, v)}{d t}=N_{1}(t, r, v) \int_{-\infty}^{+\infty} \sigma_{1}\left(v^{\prime}-v\right) \Phi_{1}^{L}\left(t, r, v^{\prime}\right) d v^{\prime} \\
& -N_{3}(t, r, v) \frac{g_{1}}{g_{3}} \int_{-\infty}^{+\infty} \sigma_{1}\left(v^{\prime}-v\right) \Phi_{1}^{L}\left(t, r, v^{\prime}\right) d v^{\prime} \\
& -N_{3}(t, r, v) \int_{-\infty}^{+\infty} \sigma_{2}\left(v^{\prime}-v\right) \Phi_{2}^{L}\left(t, r, v^{\prime}\right) d v^{\prime} \\
& +N_{6}(t, r, v) \frac{g_{3}}{g_{6}} \int_{-\infty}^{+\infty} \sigma_{2}\left(v^{\prime}-v\right) \Phi_{2}^{L}\left(t, r, v^{\prime}\right) d v^{\prime}-\frac{N_{3}(t, r, v)}{\tau_{31}} \\
& +\frac{N_{4}(t, r, v)}{\tau_{43}}+\frac{N_{6}(t, r, v)}{\tau_{63}} \\
& \frac{\partial N_{4}(t, r, v)}{d t}=\frac{N_{5}(t, r, v)}{\tau_{54}}-\frac{N_{4}(t, r, v)}{\tau_{42}}-\frac{N_{4}(t, r, v)}{\tau_{43}} \\
& \frac{\partial N_{5}(t, r, v)}{d t}=\frac{N_{6}(t, r, v)}{\tau_{65}}-\frac{N_{5}(t, r, v)}{\tau_{54}}-\frac{N_{5}(t, r, v)}{\tau_{51}} \\
& \frac{\partial N_{6}(t, r, v)}{d t}=N_{3}(t, r, v) \int_{-\infty}^{+\infty} \sigma_{2}\left(v^{\prime}-v\right) \Phi_{2}^{L}\left(t, r, v^{\prime}\right) d v^{\prime}-\frac{N_{6}(t, r, v)}{\tau_{63}} \\
& -\frac{N_{6}(t, r, v)}{\tau_{65}}-N_{6}(t, r, v) \frac{g_{3}}{g_{6}} \int_{-\infty}^{+\infty} \sigma_{2}\left(v^{\prime}-v\right) \Phi_{2}^{L}\left(t, r, v^{\prime}\right) d v^{\prime}
\end{aligned}
$$

For a non dissipative atomic system we have at any time:

$$
\sum_{i=1}^{6} N_{i}(t, r, v)=N_{D}(v)
$$

The homogeneous absorption has a lorentzian profile:

$$
\sigma_{i}(v)=\frac{\sigma_{0 i}\left(\Gamma_{i} / 2\right)^{2}}{\left(v_{0 i}-v\right)^{2}+\left(\Gamma_{i} / 2\right)^{2}}
$$

The laser profile $\Phi^{L}$ is supposed to be separable. Consequently, it is expressed as the product of the laser temporal pulse shape $\varphi(t)$ (photons/s) by the spatial distribution of the photons per unit area $\delta(r)\left(\mathrm{m}^{-2}\right)$ and the line profile $g(v)\left(\mathrm{Hz}^{-1}\right)$.

$$
\Phi^{L}(t, \vec{r}, v)=\varphi(t) \delta(\vec{r}) g(v)
$$

All numerical parameters of the REM are those used in [6] except $\sigma_{01}$ and $\sigma_{02}$ which have been recalculated in paragraph 1.1.2.1 (table 1).

\section{Flux budget : asymptotic limits and general trends}

\subsection{Upper- and asymptotic limits}

In this paragraph, we present straightforward calculations to estimate the order of magnitude of the return flux at $589 \mathrm{~nm}$ and $330 \mathrm{~nm}$, both in the weak and in the strong saturation limit. These considerations are based on simple hypotheses on the probability of absorption by the atoms. We consider that each atom can be excited with the same probability, whatever its Doppler shift might be. This implies that the sodium layer is 
excited by a laser whose spectrum covers all velocity classes i.e. by a modeless laser. To check the validity of these simple asymptotic calculations, we compare them to the results given by the Beacon and REM codes with a modeless laser.

In the strong saturation limit, we evaluate the number of photons each atom can emit at best. In the limit of the linear regime, we evaluate the asymptotic values of the return fluxes. We show that the best-case limits are coherent with the numerical values, and that the latter tend very precisely to the asymptotic limits.

\subsubsection{Strong saturation limit}

The return flux both at $589 \mathrm{~nm}$ and $330 \mathrm{~nm}$ increases with the laser power in a non-linear manner: for high laser powers, saturation strongly affects the fluorescence yield. However it is possible to give a maximal value of the fluorescence at $589 \mathrm{~nm}$ and $330 \mathrm{~nm}$ when the laser power tends to high values. In the best case, an atom shined by a laser pulse of duration $\tau_{\text {laser }}$ and excited to a level with a radiative lifetime $\tau_{b}$, can emit $1+\tau_{\text {laser }} / \tau_{b}$ fluorescence photons per laser pulse. Thus taking a $50 \mathrm{~ns}$ duration laser pulse, a strongly saturated atom can emit at best 4 photons at $589 \mathrm{~nm}$ per laser pulse $\left(\tau_{3 P_{3 / 2}}=16 \mathrm{~ns}\right)$.

Then the maximum flux available at $589 \mathrm{~nm}$ is given by:

$$
\Phi_{589}=4 \frac{S \cdot f \cdot d_{N a}}{4 \pi D^{2}}
$$

$\mathrm{S}$ is the surface of the laser spot, $f$ is the repetition rate of the laser, $d_{N a}$ is the surface density of the sodium layer $\left(=410^{13}\right.$ atoms $/ \mathrm{m}^{2}$ ). $D$ is the altitude of the sodium layer.

For the transition at $330 \mathrm{~nm}$, an atom can undergo at best two spontaneous decays from the $4 \mathrm{D}_{5 / 2}$ state per laser pulse $\left(\tau_{4 D_{5 / 2}}=54 \mathrm{~ns}\right)$. From this state, the atom can decay to the $4 \mathrm{P}_{3 / 2}$ level with a probability of $1 / 3$, and emit a photon at 330 $\mathrm{nm}$ with a probability of $1 / 9$. But in this latter case, since the radiative lifetime of the $4 \mathrm{P}_{3 / 2}$ level is larger than the laser pulse duration, the atom cannot be promoted again to the $4 \mathrm{D}_{5 / 2}$ level. Therefore the maximum return flux at $330 \mathrm{~nm}$ is simply given by:

$$
\Phi_{330}=\frac{1}{9} \frac{S \cdot f \cdot d_{N a}}{4 \pi D^{2}}
$$

Note that the maximal flux at both $589 \mathrm{~nm}$ and $330 \mathrm{~nm}$ evolves linearly with the size of the laser spot, and is independent from the laser intensity.

\subsubsection{Weak saturation limit}

Here we evaluate the asymptotic behaviour of the $589 \mathrm{~nm}$ return flux in the weak saturation limit, i.e. in the linear regime. For that, we adopt a Beer-Lambert type approach.

We consider a single sodium atom initially in the $3 \mathrm{~S}_{1 / 2}$ level. The number of photons seen by the atom per second and corresponding to its velocity class is:
$\varphi_{589}=\frac{\bar{I}_{1}}{S} \frac{\Gamma_{1}}{\delta v_{1}} \frac{1}{\hbar \omega_{1}}$

where $\Gamma_{1}$ is the homogeneous width of the $3 \mathrm{~S}_{1 / 2} \rightarrow 3 \mathrm{P}_{3 / 2}$ transition, $\delta v_{1}$ is the spectral width of the first laser, $\bar{I}_{1}$ the average laser power at $589 \mathrm{~nm}$ and $\omega_{1}$ the angular frequency at $589 \mathrm{~nm}$. (Similar conventions are taken for the transition $3 \mathrm{P}_{3 / 2} \rightarrow 4 \mathrm{D}_{5 / 2}$ at $569 \mathrm{~nm}$, with the subscript 2.) We consider here that the spectral width of the laser is larger than the homogeneous width, which is always the case for tens of ns laser pulses. We also consider that the laser width is narrower than the Doppler width $\Gamma_{1}^{D}$ of the transition.

The probability of excitation (number of excitations per second) of this atom can be written as:

$p=\sigma_{01} \varphi_{589}$

The number of atoms shined by the laser is:

$n_{N a}=S . d_{N a} \frac{\delta v_{1}}{\Gamma_{1}^{D}}$

Therefore the return flux at $589 \mathrm{~nm}$ is:

$\Phi_{589}=\frac{n_{N a}}{4 \pi D^{2}} p=\sigma_{01} \frac{d_{N a}}{4 \pi D^{2}} \frac{\bar{I}_{1}}{\hbar \omega_{1}} \frac{\Gamma_{1}}{\Gamma_{1}^{D}}$

Note that in the linear regime, the one-photon excitation process is proportional to the laser intensity, and is independent from the size of the laser spot.

We estimate now the return flux at $330 \mathrm{~nm}$ in the weak saturation limit. For simplicity reasons, we consider that both lasers cover exactly the Doppler widths of the transitions.

During a laser pulse, the number of sodium atoms excited to the $3 \mathrm{P}_{3 / 2}$ level is:

$n_{3 P_{3 / 2}}=\sigma_{01} \frac{\bar{I}_{1}}{f} \frac{\Gamma_{1}}{\Gamma_{1}^{D}} \frac{1}{\hbar \omega_{1}} d_{N a}$

For one of these atoms, the number of photons at $569 \mathrm{~nm}$ per laser pulse corresponding to their velocity class is:

$\varphi_{569}=\frac{1}{f} \frac{\bar{I}_{2}}{S} \frac{\Gamma_{2}}{\Gamma_{2}^{D}} \frac{1}{\hbar \omega_{2}}$

The probability of excitation to the $4 \mathrm{D}_{5 / 2}$ level, per atom in the $3 \mathrm{P}_{3 / 2}$ level, is therefore:

$p=\frac{\tau_{3 P_{3 / 2}}}{\tau_{\text {laser }}} \sigma_{02} \varphi_{569}$

The prefactor comes from the fact that the laser pulse is longer than the radiative lifetime of the intermediate level $3 \mathrm{P}_{3 / 2}$, which reduces the effective probability of an atom in the intermediate level to absorb a photon at $569 \mathrm{~nm}$ within its lifetime.

Finally, the return flux at $330 \mathrm{~nm}$ is: 


$$
\Phi_{330}=\frac{1}{9} \frac{d_{N a}}{4 \pi D^{2}} \frac{\tau_{3 P_{3 / 2}}}{\tau_{\text {laser }}} \frac{1}{f} \sigma_{01} \sigma_{02} \frac{\bar{I}_{1}}{\hbar \omega_{1}} \frac{\bar{I}_{2}}{\hbar \omega_{2}} \frac{\Gamma_{1}}{\Gamma_{1}^{D}} \frac{\Gamma_{2}}{\Gamma_{2}^{D}} \frac{1}{S}
$$

The probability to populate the $4 \mathrm{D}_{5 / 2}$ is proportional to both the population of the $3 \mathrm{P}_{3 / 2}$ level and the intensity of the laser at 569 $\mathrm{nm}$. When both laser intensities are taken equal, this probability evolves as the square of the laser power. Note also that the return flux at $330 \mathrm{~nm}$ in the regime of weak saturation, is proportional to the inverse of the spot size and to the inverse of the repetition rate of the laser.

\subsubsection{Numerical estimations}

\subsubsection{Return flux vs. laser intensity}

Here, we consider the case of two modeless lasers, operating at $17 \mathrm{kHz}$ repetition rate. The pulse duration is fixed at $50 \mathrm{~ns}$ and the time shape is a square function. The spectral width of the laser at $589 \mathrm{~nm}$ (resp. $569 \mathrm{~nm}$ ) is set to $3 \mathrm{GHz}$ (resp. 1GHz). Both lasers have the same average intensity $\bar{I}$. The size of the laser spot is $0.5 \mathrm{~m}^{2}$. We compare the numerical data from both Beacon and the REM, with the asymptotic laws obtained in the previous paragraph.

In the limit of weak laser power, we have:

- $\Phi_{589}=4.310^{5} . \bar{I}$ photons $/ \mathrm{s} / \mathrm{m}^{2}$ and $\bar{I}$ given in $\mathrm{W}$,

- $\Phi_{330}=8.110^{2} . \bar{I}^{2}$ photons $/ \mathrm{s} / \mathrm{m}^{2}$ and $\bar{I}$ given in $\mathrm{W}$.

In the strong laser power limit, we have:

- $\Phi_{589}=1.310^{7}$ photons $/ \mathrm{s} / \mathrm{m}^{2}$,

- $\Phi_{330}=3.710^{5}$ photons $/ \mathrm{s} / \mathrm{m}^{2}$.

Note on Fig. 5 the good agreement between the asymptotic and "best case" limits, and the results of the Beacon and REM codes, both in the weak and in the strong saturation regime. It is also worth noting that the ratio between the return flux at 589 $\mathrm{nm}$ and $330 \mathrm{~nm}$ is at least equal to $\sim 30$ (in the strong saturation limit) and increases towards the linear regime.

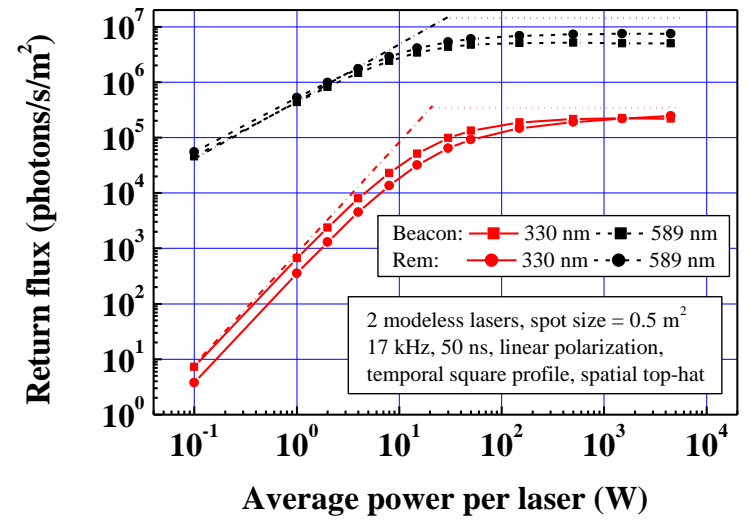

Fig. 5. Return flux of the PLGS with respect to the average laser power. Scatter: Beacon and REM data; dotted lines: "best-case" limits, dash-dot lines: asymptotes in the linear regime.

\subsubsection{Return flux vs. spot size}

In this paragraph, we study the asymptotic behaviours in the return flux vs. laser spot size representation. We evidence that the return flux for the one-photon excitation (monochromatic LGS) exhibits a very different behaviour from the return flux of the PLGS. We still consider the case of two modeless lasers, operating at $17 \mathrm{kHz}$. The (square) pulse duration is set at $50 \mathrm{~ns}$. The average power for each laser is $15 \mathrm{~W}$.

In the small spot size limit (i.e. in the strong saturation limit), the "best-case" expressions for the flux at $589 \mathrm{~nm}$ and $330 \mathrm{~nm}$ are deduced from the previous relations. We obtain:

- $\Phi_{589}=2.710^{7} . S$ photons $/ \mathrm{s} / \mathrm{m}^{2}$ and $S$ given in $\mathrm{m}^{2}$,

- $\Phi_{330}=7.410^{5} . S$ photons $/ \mathrm{s} / \mathrm{m}^{2}$ and $S$ given in $\mathrm{m}^{2}$.

In the large spot size limit (i.e. in the weak saturation limit), the asymptotic expressions for the flux at $589 \mathrm{~nm}$ and $330 \mathrm{~nm}$ give respectively:

- $\Phi_{589}=6.410^{6}$ photons $/ \mathrm{s} / \mathrm{m}^{2}$,

- $\Phi_{330}=\frac{9.110^{4}}{S}$ photons $/ \mathrm{s} / \mathrm{m}^{2}$ and $S$ given in $\mathrm{m}^{2}$.

As can be seen on fig. 6, the agreement between both models is still good for both wavelengths in the small and large spot limit. Note that the return flux at $589 \mathrm{~nm}$ tends to its asymptotic value for large spot sizes. This is not the case for the return flux of the PLGS, which exhibits a maximum. This comes from the fact that the return flux at $330 \mathrm{~nm}$ is limited by two phenomena: the saturation of the atom on the side of small spot sizes, and the intrinsic non-linearity of the two-photon excitation process for large spot sizes.

\subsection{Figure of merit of the polychromatic laser guide star}

The representation of the return flux vs. spot size is also pertinent to determine the figure of merit of the PLGS. The spot size is determined by several parameters: the quality of the laser beam, the diameter and quality of the laser projector, the Fried parameter $\mathrm{r}_{0}$, among others. The solid vertical line on fig. 6 represents the diffraction limited spot through a $50 \mathrm{~cm}$ projector. The precision on the measurement of the tip-tilt is linked to two parameters: the intensity of the available return flux, and the size of the image on the tip-tilt sensor (a CCD camera or a 4-quadrant photodiode for example). Concerning the first parameter, for a given image size (in a X-Y representation), the precision in the $\mathrm{X}$ direction $\Delta X$ is ideally determined by the photon noise, i.e. it is proportional to $1 / \sqrt{\Pi}, \Pi$ being the number of photoelectrons detected by the AO system per acquisition frame. $\Pi$ is simply proportional to the return flux $\Phi_{330}$. The dependence of the precision of the tip-tilt measurement with the size of the image of the fluorescence is not so straightforward. The point is that Beacon uses the laser surface density as an input, assuming implicitly 
top-hat spatial profiles. However in reality the fluorescence spot does usually not match exactly the laser spot in the mesosphere, the reason being that on one side, saturation tends to increase the apparent width of the spot and that on the other side, the nonlinearity of the two-photon process in the case of the PLGS tends to reduce the size of the fluorescence spot compared to the size of the laser spot. Therefore in the general case, when $\Pi$ is constant, the precision in the $\mathrm{X}$ direction $\Delta X$ is proportional to the diameter of the spot, i.e. to $\sqrt{\Sigma}$ where $\Sigma$ is the area of the fluorescence spot, which usually differs from $\mathrm{S}$, the area of the laser spot.

The precision on the tip-tilt evolves as:

$$
\Delta X \Delta Y \propto \Sigma / \Phi_{330}
$$

Minimizing this ratio leads to the optimal PLGS configuration in terms of laser characteristics (power, polarization, spectrum, temporal shape...) and spot size at the mesosphere. Therefore we can define the figure of merit for the PLGS as $\Phi_{330} / \Sigma$. The best solution in terms of lasers should maximize this parameter. The difficulty arises from the knowledge of the fluorescence spot: the propagation of the laser beam to the mesosphere leads to wavefront distortion and speckle effects that are usually difficult to control.

However for illustration purposes, it is interesting to consider the ideal case where the laser beam has a top-hat profile in the mesosphere. In that case, since the power density of both lasers is constant over the area shined by the lasers, the fluorescence spot matches exactly the laser spot and there is a simple proportionality between $\Sigma$ and S. Therefore the figure of merit of the PLGS evolves as: $\Phi_{330} / \mathrm{S}$. The best situation corresponds to a return flux as high as possible and to a laser spot as small as possible. The dashed curve in fig. 6 represents the figure of merit for the case discussed in the previous paragraph. The vertical scale is logarithmic, with arbitrary units. Here the best case (where the uncertainty on the position of the chromatic component of the PLGS is the smallest) corresponds to the limit of high saturation that is to say towards small laser spots.

The optimization of the figure of merit in the real case of gaussian laser modes distorted by atmospheric propagation would constitute an interesting discussion, exceeding the frame of this paper.

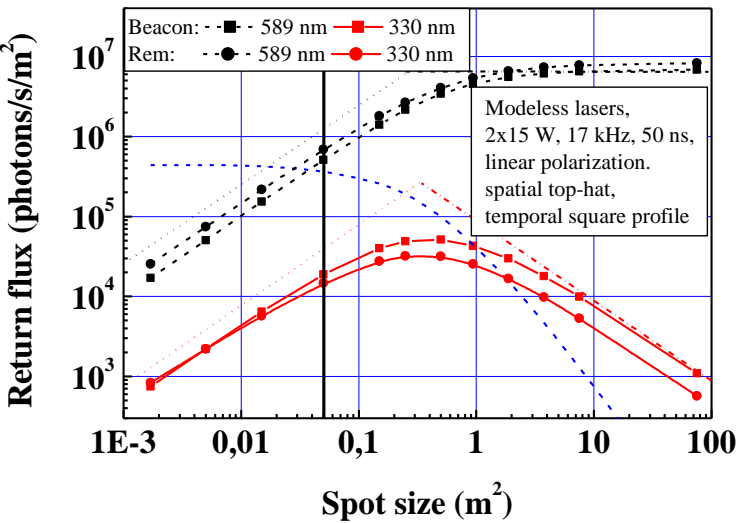

Fig. 6. Return flux of the PLGS with respect to the spot size. Scatter: Beacon and REM data; dotted lines: "best-case" limits, dash-dot lines: asymptotes in the linear regime. The dash curve represents the figure of merit of the PLGS process in arb. units (see $\S 2.2$ ). The solid vertical line at $0.05 \mathrm{~m}^{2}$ corresponds to a diffraction limited spot through a $50 \mathrm{~cm}$ projector.

\subsection{Influence of laser parameters: straightforward aspects} In this paragraph, we present tendencies in the behaviour of the return flux when specific experimental parameters are varied. This is of particular importance when one wants to spare calculation time: we show that from the knowledge of a single curve (return flux vs. laser intensity or vs. laser spot size) for a given set of laser parameters, it is possible to deduce without any further calculation the return flux curve when one (or some) of the laser parameters is (are) varied. This is quite useful when starting from a requested return flux and laser spot size, one wants to define the corresponding laser solution (in terms of mean power, rep. rate, etc... ): this possibility is illustrated in the final conclusion of this paper.

The return flux depends on many parameters: the laser peak power, the repetition rate, the size of the laser spot, etc. However, these parameters are somehow interdependent, and this can be used to avoid additional simulations and to save computing time. We detail here the relations between the laser parameters, and we show how this can be used to predict different cases.

\subsubsection{Influence of the repetition rate}

For a given peak power intensity, the return fluxes $\Phi_{589}$ and $\Phi_{330}$ are proportional to the number of atoms excited (i.e. to the surface of the spot $S$ ) and to the laser rep. rate $f$. However there is no proportionality between the laser peak intensity $I_{\text {peak }}$ and the return flux. The latter can be written as:

$\Phi=S . f . F\left(I_{\text {peak }}\right)$

where $F$ is an unknown nonlinear function.

We also have $I_{\text {peak }}$ proportional to $\frac{\bar{I}}{S . f}$. Therefore 
$\Phi=S . f \cdot F\left(\frac{\bar{I}}{S . f}\right)$

We calculate the return flux when the repetition rate is increased by a factor $k$, for given spot size and average power. We have:

$$
\begin{aligned}
\Phi(S, k \cdot f, \bar{I}) & =S . k . f . F\left(\frac{\bar{I}}{S . k \cdot f}\right) \\
& =\Phi(k . S, f, \bar{I})
\end{aligned}
$$

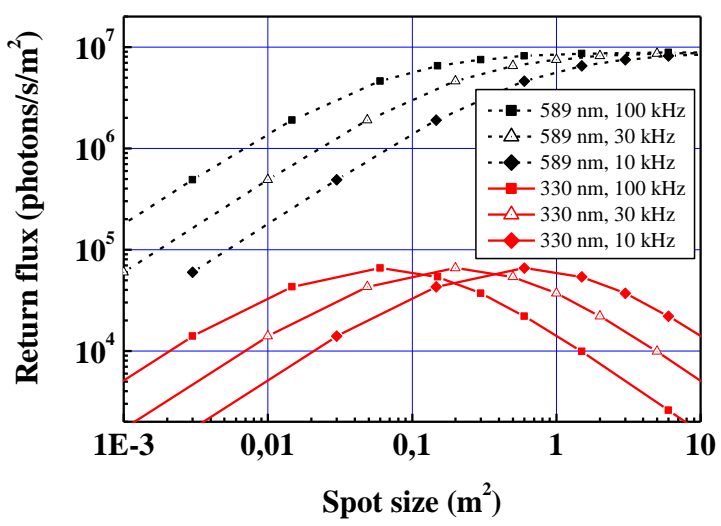

Fig. 7. Return flux of the PLGS given by Beacon, with respect to the spot size for different rep. rates. The lasers average power is constant; (Laser power = $2 * 18 \mathrm{~W}$, circular polarization, modeless laser, $80 \mathrm{~ns}$ gaussian time shape, tophat spatial profile).

This means that in a graph giving the return flux vs. spot surface, an increase of the rep. rate by a factor of $k$, results in a homothetic transformation on the horizontal scale by a factor of $1 / k$ (fig. 7). This is of particular importance if one considers the figure of merit of the PLGS: in the saturation regime and for a constant laser power, it is desirable to increase the repetition rate to shift the flux curves to small spot sizes, resulting in increasing the figure of merit.

We also have:

$\Phi(S, k \cdot f, \bar{I})=k \cdot \Phi(S, f, \bar{I} / k)$

which means that in a graph plotting the return flux vs. laser power, an increase of the rep. rate by a factor of $k$, results in a homothetic transformation on the horizontal scale by a factor of $k$, and on a vertical scale by a factor of $k$ (fig. 8).

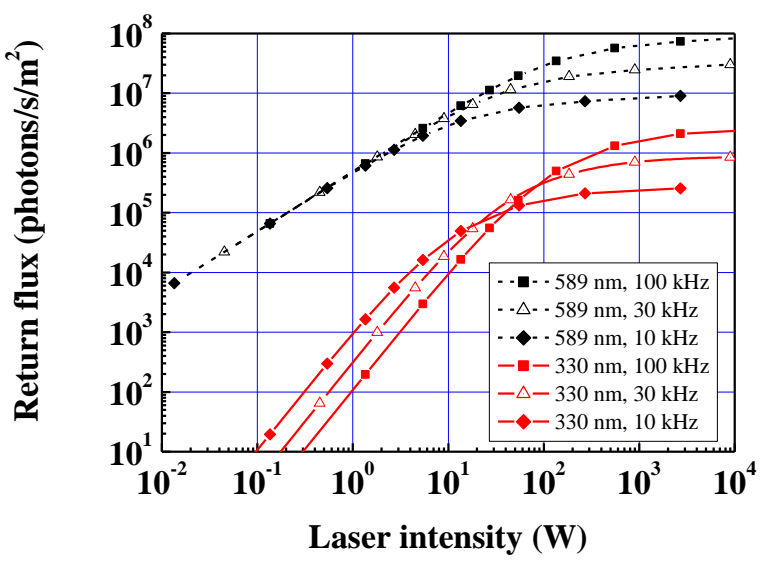

Fig. 8. Return flux of the PLGS given by Beacon, with respect to the laser intensity (per laser) for different repetition rates (spot size $=0.5 \mathrm{~m}^{2}$, circular polarization, modeless laser, $80 \mathrm{~ns}$ gaussian time shape, top-hat spatial profile).

\subsubsection{Influence of the total laser power}

We consider now the case where the average laser power is increased by a factor $k$, all other parameters being unchanged. Then the return flux is:

$$
\begin{aligned}
\Phi(S, f, k . \bar{I}) & =S \cdot f \cdot F\left(\frac{k \cdot \bar{I}}{S \cdot f}\right) \\
& =k \cdot(S / k) \cdot f \cdot F\left(\frac{\bar{I}}{(S / k) \cdot f}\right) \\
& =k \cdot \Phi(S / k, f, \bar{I})
\end{aligned}
$$

This means that in a return flux vs. spot size plot, an increase of the laser power by a factor of $k$ results in a homothetic shift by a factor of $k$ along both the horizontal and the vertical axis (see fig. 9).

Interestingly, the graph linking the return flux to the spot size curve is unchanged on the left side of the plot. This simply means that in the strong saturation limit, increasing the laser power does not increase the figure of merit of the PLGS (all other parameters being constant).

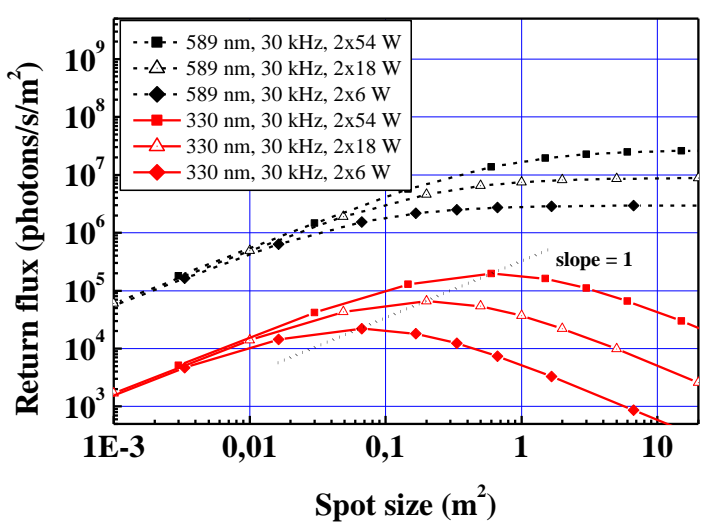


Fig. 9. Return flux of the PLGS given by Beacon, with respect to the spot size for different laser powers (rep. rate $=30 \mathrm{kHz}$, circular polarization, modeless laser, $80 \mathrm{~ns}$ gaussian time shape, top-hat spatial profile).

\subsubsection{Influence of the laser spot size}

Finally we consider the case where the spot area is increased by a factor of $k$, all parameters being constant. In that case, the return flux is given by:

$$
\Phi(k . S, f, \bar{I})=k . S . f . F\left(\frac{\bar{I}}{k . S . f}\right)=k . \Phi(S, f, \bar{I} / k)
$$

This indicates that in a return flux vs. laser intensity plot, an increase of the laser spot size by a factor of $k$ results in a homothetic shift by a factor of $k$ along both the horizontal and the vertical axis (fig. 10).

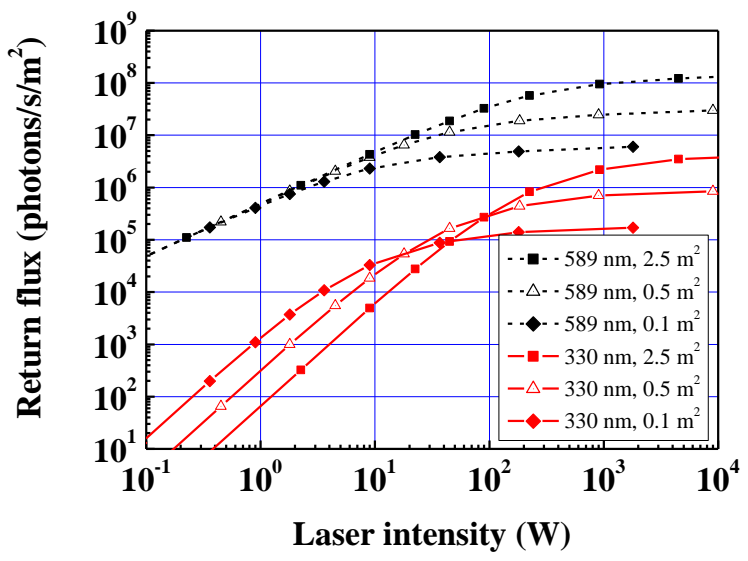

Fig. 10. Return flux of the PLGS given by Beacon, with respect to the laser intensity (per laser) for different laser spot sizes (rep. rate $=30 \mathrm{kHz}$, circular polarization, modeless laser, $80 \mathrm{~ns}$ gaussian time shape, top-hat spatial profile).

\section{Choice of the laser system}

As we mentioned it before, we restrict this study to the case of tens of nanoseconds laser pulses, with a repetition rate of few tens of $\mathrm{kHz}$. In this paragraph, we compare four types of lasers: a single mode laser, a phase-modulated laser, a modeless and a multimode laser.

\subsection{Single-mode vs. phase modulated lasers}

The return flux of the PLGS is controlled by two limitations: on one side, the two-photon absorption is a non-linear phenomenon and requires high laser intensities. On the other side, the saturation limits the fluorescence yield for high peak intensities. A nice way to reduce the saturation is to dispatch the laser intensity over as many sodium velocity classes as possible, i.e. to broaden the laser spectrum. This enables to decrease the saturation for small spot sizes and to improve the figure of merit (see $\S 2.2$ ). Phase modulation (PM) of the laser beam is an efficient way to add sidebands to the laser spectrum and to improve the overlap with the atomic spectral lines. The phase modulation relies on the electro-optic (EO) effect. A sinusoidal voltage is applied to the crystal, resulting in a periodic modulation of the linear phase of the laser field at the output of the crystal. This time-dependent phase leads to additional lines in the power spectrum of the laser field. (Note that to increase the spectral width additional modulators can be inserted in series on the laser beam path.) The frequency shift of the additional spectral lines is a linear combination of the frequencies applied to the EO modulators, whereas their intensity is dictated by non-trivial behaviours, except when a single phase-modulation is applied: in this case the spectrum gets simpler and is described by Bessel functions. The instantaneous phase shift experienced by the light across a single EO modulator is simply: $\Phi(t)=\frac{M}{v} \sin (v t)$, where $M$ is called the modulation intensity (in $\mathrm{MHz}$ ), and $v$ is the frequency applied to the modulator (in $\mathrm{MHz}$ ).

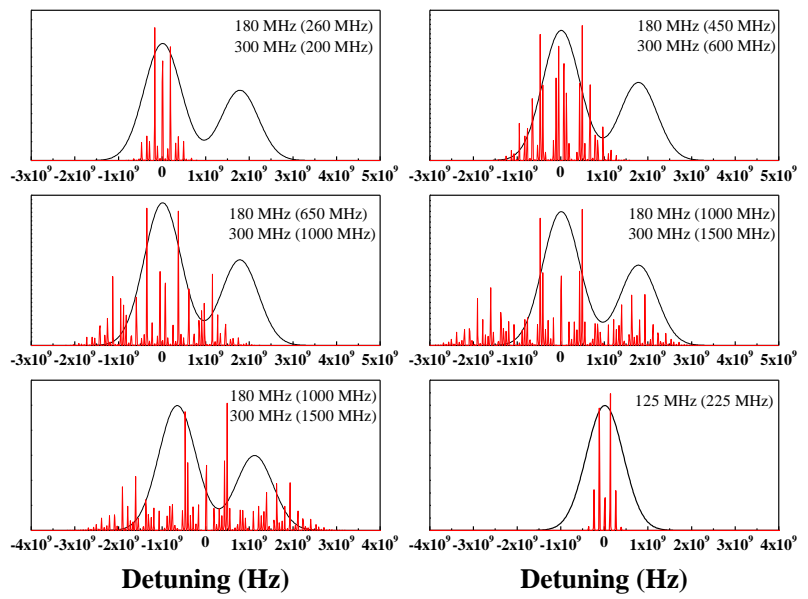

Fig. 11. Spectra corresponding to different phase modulations with respect to the hyperfine-Doppler lines of the transitions at 589 and $569 \mathrm{~nm}$. The bottom right curve is the spectrum for a laser at $569 \mathrm{~nm}$, modulated at $125 \mathrm{MHz}$ with an intensity equal to $225 \mathrm{MHz}$. All other graphs concern the double phase modulation of the first laser. All PM frequencies are 180 and $300 \mathrm{MHz}$. The associated intensities are given in parentheses. Note that all spectra are centred on the $\mathrm{F}=2$ line of the $\mathrm{D}_{2}$ line, except the bottom left one, centred on the $\mathrm{D}_{2}$ line.

The Beacon code enables to introduce up to three phase modulations on each of the lasers. First, we restrict ourselves to a single phase modulation on the laser at $569 \mathrm{~nm}$, and a double modulation on the laser at $589 \mathrm{~nm}$, as this was the case during the PASS-2 campaign [18,23]. We consider the case of $50 \mathrm{~ns}$ pulses, $2 * 15 \mathrm{~W}$ average power, linear polarization and $17 \mathrm{kHz}$ repetition rate. The time profiles are gaussian and the spatial one is a top-hat. Several configurations have been tested, their spectra are plotted on fig. 11, and the numerical results are presented on fig. 12. 


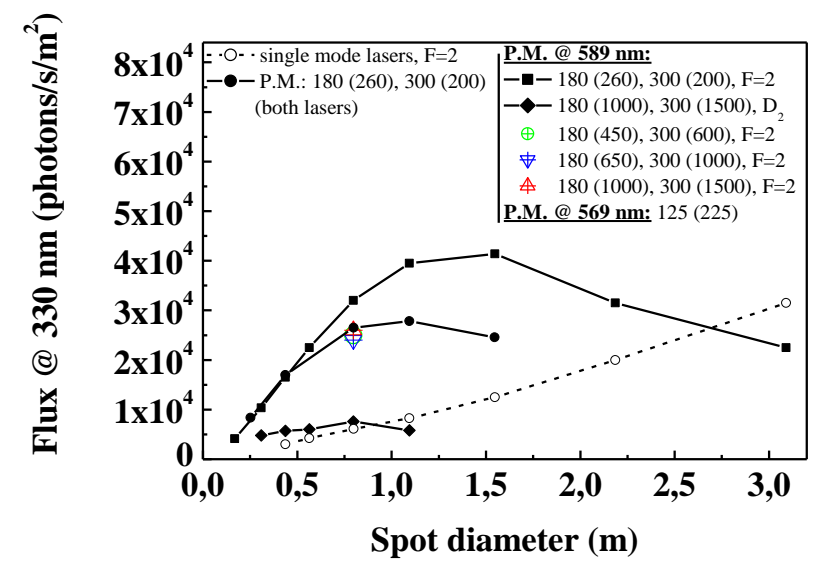

Fig. 12. Return flux at $330 \mathrm{~nm}$ given by Beacon, with respect to the laser spot diameter for different phase modulation functions. Laser parameters: $2 * 15 \mathrm{~W}$, $50 \mathrm{~ns}$, linear polarization, gaussian time profile, spatial top-hat. All PM frequencies are 180 and $300 \mathrm{MHz}$. The associated intensities are given in parentheses. All values are given in $\mathrm{MHz}$. The mention $\mathrm{D}_{2}$ or $\mathrm{F}=2$ indicates the position of the central frequency of the phase modulation.

Generally speaking, phase modulation shows an evident gain over single mode lasers, which tend to reach their maximal return flux for very large spot sizes. Concerning the choice of the phase modulation, the best results in terms of return flux correspond to a laser centred on $\mathrm{F}=2$ with a relatively small spectral width. When the spectral width of the first laser is increased to excite all velocity classes of the $\mathrm{D}_{2}$ line, the return flux at $330 \mathrm{~nm}$ is lower. The difficulty associated with the phase modulation is that in principle any frequency line on the first laser should to have its counterpart on the second one: a velocity class excited by the first laser needs also to be reached by the second one for an efficient population of the $4 \mathrm{D}_{5 / 2}$ level. If one wants to cover the whole $\mathrm{D}_{2}$ line, this would require extremely complicated phase modulation functions. Moreover from an experimental point of view, any jitter on the laser spectra has an important impact on the efficiency of the twophoton transition.

To check the possible benefits of overlapping the two laser spectral lines, we present the results obtained by applying the same PM on both lasers. We choose to restrict the excitation of the first transition to the $F=2$ line of the $D_{2}$ transition and we use the following parameters: the modulation frequencies are $180 \mathrm{MHz}$ and $300 \mathrm{MHz}$ and the respective intensities are 260 $\mathrm{MHz}$ and $200 \mathrm{MHz}$. The results are presented also on fig. 12. But surprisingly, we observe that in the case of identical phase modulations on both lasers, the plot return flux at $330 \mathrm{~nm}$ vs. spot diameter is not increased: contrary to what could be expected, the maximum return flux is smaller. We also can note that the maximum of the fluorescence curve is shifted to the small spots, but at the expense of lowering the return flux.

\subsection{Modeless laser}

The benefits brought by the phase modulation over the single mode operation can be increased by using a modeless laser covering all velocity classes, and for which the problem of matching the frequency lines of both lasers is automatically solved by the continuous nature of the spectrum. As a proof of demonstration, we focus on the laser situations proposed in [5]. We ran the Beacon code for the different situations proposed in the publication. In the case called ELPOA 1, the lasers are both phase-modulated, their mean power at the mesosphere is $18 \mathrm{~W}$, the repetition rate is $15 \mathrm{kHz}$, the pulse duration is $50 \mathrm{~ns}$ and the lasers are linearly polarized. In both cases ELPOA 2 and 3, the laser parameters are: $18 \mathrm{~W}$ average power, $30 \mathrm{kHz}$ repetition rate, (gaussian) 80 ns pulse duration and circular polarizations. The spatial mode of the lasers is a top-hat; the difference being that in ELPOA 2, the lasers are phase-modulated, whereas they are modeless in the ELPOA 3 case. Since [5] does not explicit the phase modulation function, we took the same phase modulation for ELPOA 1 and ELPOA 2: $180 \mathrm{MHz}(260 \mathrm{MHz})$ and $300 \mathrm{MHz}(200 \mathrm{MHz})$ for the first laser and $125 \mathrm{MHz}(250$ $\mathrm{MHz}$ ) for the second one [18]. The results given by the Beacon code are plotted in fig. 13. Concerning the difference between ELPOA 1 and 2, we observe that the flux curves of ELPOA 1 are shifted to the right compared to ELPOA 2: this corresponds to the fact that the ELPOA 1 case is more saturated than the ELPOA 2 case (smaller repetition rate and shorter laser pulse). In ELPOA 3, the value of the maximal flux at $330 \mathrm{~nm}$ is increased by a factor of $\sim 2$. Therefore the figure of merit, as defined in $\$ 2.2$ is improved in the case of the modeless laser, which appears naturally as the best solution for the PLGS [24].

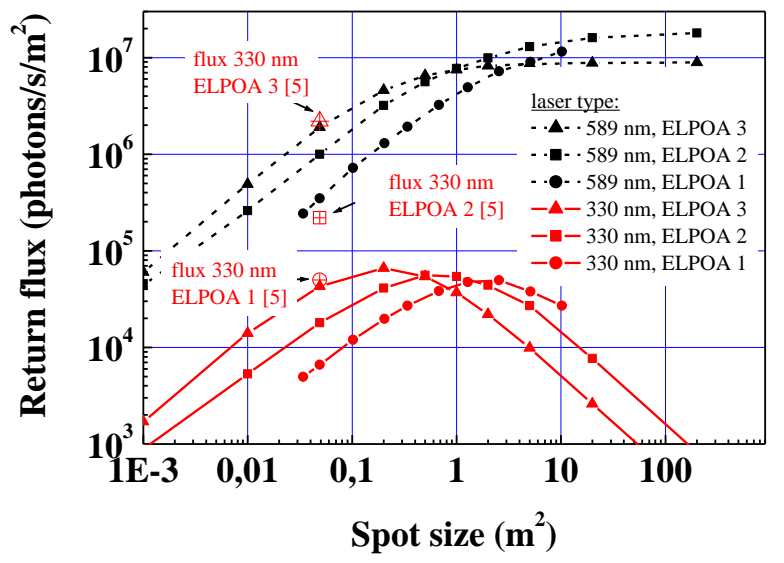

Fig. 13. Return fluxes at $589 \mathrm{~nm}$ and $330 \mathrm{~nm}$ for the cases treated in [5]. The red scatter data are the numerical values of the flux given in the paper. In this work we took the laser characteristics of ELPOA 1 : circle, ELPOA 2 : square, and ELPOA 3 : triangle. See text and conclusion for details.

\subsection{Multimode laser}

To illustrate the benefits of the modeless laser vs. single-mode or phase-modulated lasers, we can represent the modeless laser as the limit of multimode lasers, when the free spectral range becomes narrower than the homogeneous linewidth of the transitions. In this paragraph, we study the influence of the total number of laser modes in the absorption line by changing the free spectral range of the lasers. Both the modified version of 
the Beacon code and the REM can account for multimode spectra but for simplicity reasons we only present data obtained from the REM; for calibration, a few points have been also run by Beacon and are consistent with the REM results. In this case we consider $2 * 15 \mathrm{~W}$ multimode lasers (linear polarization, 50 ns pulses, $17 \mathrm{kHz}$ rep. rate) and we vary the free spectral range between $2 \mathrm{MHz}$ and $1 \mathrm{GHz}$. Recall that the first value describes the modeless laser and the second one a single mode laser. Both lasers (at $589 \mathrm{~nm}$ and $569 \mathrm{~nm}$ ) have the same FSR. The results are presented on fig. 14.
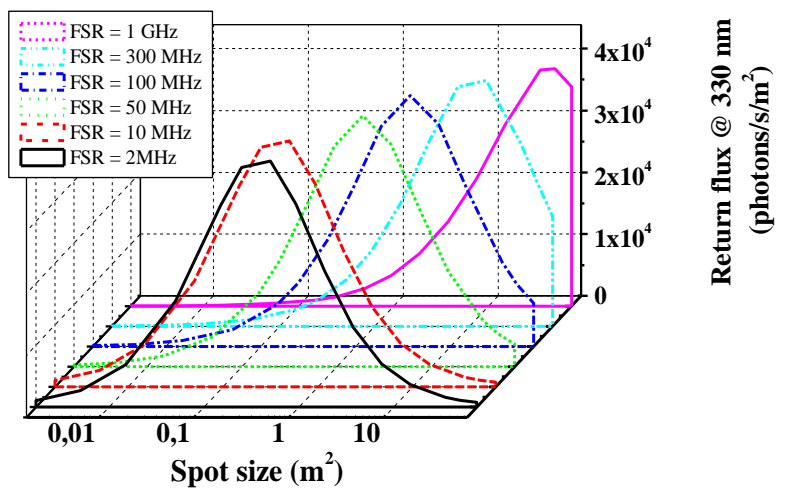

Fig. 14. Return flux at $330 \mathrm{~nm}$ vs. spot size for different values of the free spectral range (REM code).

It is worth noting that decreasing the FSR of the lasers simply shifts the curve return flux vs. spot size to the small spots (this is also the behaviour we obtain for the monochromatic LGS at $589 \mathrm{~nm}$ ). The maximum return flux is weakly affected by the value of the FSR, but in terms of maximizing the figure of merit of the PLGS, it is desirable to decrease the spot size without decreasing the return flux. This is achieved by decreasing the saturation (i.e. by increasing the density of laser modes), the asymptotic limit being the modeless laser which appears as the optimal solution for the PLGS.

\section{Optimization of the laser system}

In the last part, we test the influence of several parameters on the return flux of the PLGS, the idea being to improve the figure of merit of the PLGS in terms of both increasing the return flux and minimizing the spot size. We discuss here successively the influence of the temporal shape of the laser pulse (gaussian vs. square), the spatial mode (gaussian vs. tophat), the polarization (circular vs. linear), the spectral shape of the first laser, the pulse duration (50 ns vs. $80 \mathrm{~ns}$ ), the time delay between the laser pulses, and the repartition of the total intensity between the two lasers.

\subsection{Influence of the temporal laser profile}

Beacon and REM codes accept both options: a square or a gaussian time profile. For the Beacon code the option is set in the data input file: the square function is asymptotically approximated by hypergaussian profiles. In fig. 15 , we compare the return fluxes between the gaussian and the square profiles, all other parameters being constant.

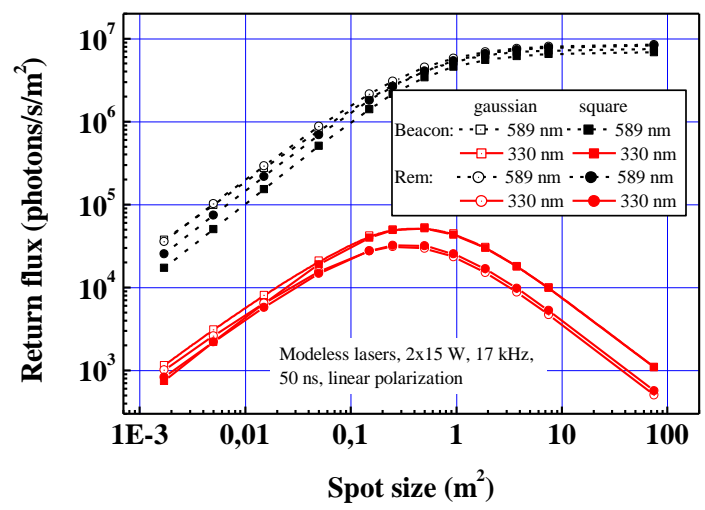

Fig. 15. Comparison of the return flux for square and gaussian time profiles (Beacon and REM codes).

Both temporal time shapes show a good qualitative agreement, the difference does not exceed $30 \%$ in the limit of strong saturation. Note also that the slope of the return flux vs. surface for small spots is strictly 1 for the square profile. The return flux for gaussian pulses is a little bit increased, compared to the square pulses: this comes from the fact that due to the wings of the gaussian function, the effective duration of the laser pulse seen by the atom is longer than in the case of square pulses. Therefore the maximum number of photons an atom can emit during a laser pulse is increased and the return flux is increased consequently.

\subsection{Influence of the spatial laser mode}

The Beacon code takes the peak power density of the laser (in $\mathrm{W} / \mathrm{cm}^{2}$ per pulse) as an input variable. Therefore it implicitly assumes a top-hat distribution of the laser intensity. In reality, the situation is different, since the laser beams are usually gaussian at the ground, and can be distorted after propagation through the atmosphere, depending on the extension of the laser beam with respect to the Fried parameter $r_{0}$. For simplicity reasons, we assume that both laser beams exhibit a gaussian mode at the mesosphere. We also assume that these modes have the same spatial extension. In the paragraph, we study the difference between the top-hat approximation, and the gaussian one. To take into account the gaussian profile, we sample the gaussian function by a step-like function. The Beacon code is ran for each discrete step and the set of return flux values is used to evaluate the return flux for the gaussian case. To compare both cases, we define the relative widths of the top hat and the gaussian mode accordingly to Siegman: the radius of the top-hat profile is equal to $w / \sqrt{2}, w$ being the waist of the gaussian function [25].

As plotted in fig. 16, the agreement between the gaussian shape and the top-hat is good in the linear regime (large spot sizes). 
However, in the saturated regime, the gaussian shape leads to a large increase in the return flux, up to a factor of 4 for very saturated cases. Note also that the maximum of the curve $\Phi_{330}(S)$ is shifted to the small spot sizes by a factor of 2 , and the maximum flux is slightly higher in the gaussian than in the top-hat case. This behaviour is due to the fact that the gaussian case is less saturated, since the spatial extension of the beam is not limited, contrary to the top-hat profile. Note that in the case of the gaussian spatial mode, the effective size of the fluorescence spot is no more equal to the laser spot size defined by the horizontal axis. Note also that it is possible experimentally to generate top-hat spatial profiles, but at the expense of loosing a substantial laser power.

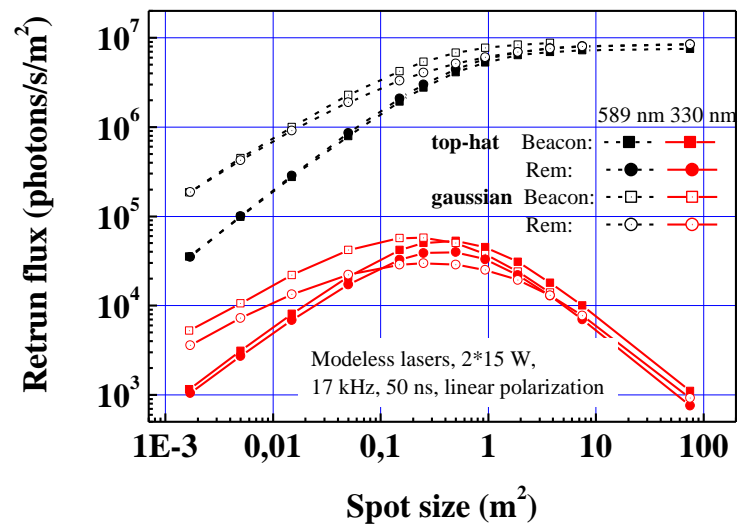

Fig. 16. Comparison of the return flux for square and gaussian spatial profiles (Beacon and REM codes). Both time profiles are gaussian.

\subsection{Influence of the polarization}

Another possibility of the BEACON code is to set the polarization of the laser beams. This is of particular interest since the hyperfine structure is taken into account and the excitation probability of the sodium atom can be optimized according due to the values of the Clebsch-Gordan (CG) coefficients. For instance for the excitation of the $3 \mathrm{~S}_{1 / 2} \rightarrow 3 \mathrm{P}_{3 / 2}$ transition, the hyperfine transition $\mathrm{m}_{\mathrm{F}}=2 \rightarrow \mathrm{m}_{\mathrm{F}}$, $=3$ has the highest CG coefficient. This argument has been studied in detail in [17] and a substantial gain is obtained when circular polarization is used, instead of linear. Note that to take full advantage of this, one needs to use rather long laser pulses to accumulate the sodium atoms into large $\mathrm{m}_{\mathrm{F}}$ states.

For the two photon excitation, we investigated the benefit of circular vs. linear polarization by comparing both cases, for both phase modulated and modeless lasers. The results given by the Beacon code are plotted in fig. 17.

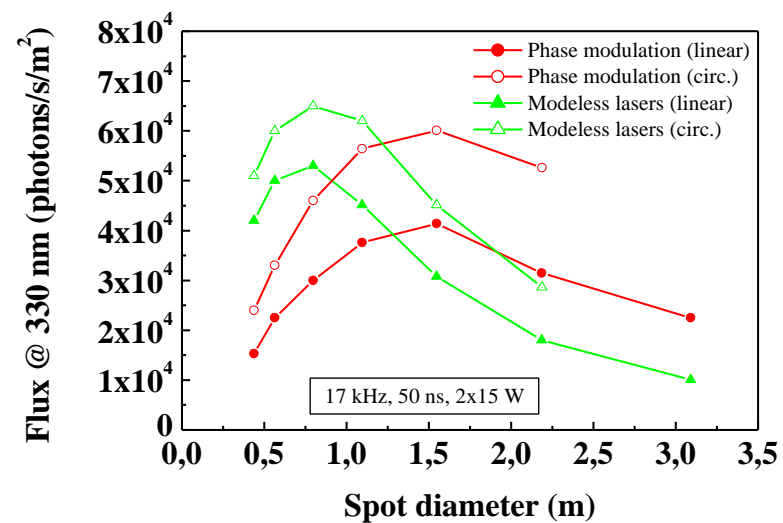

Fig. 17. Evolution of the return flux at $330 \mathrm{~nm}$ with respect to the spot diameter. Both modeless and PM lasers are presented with linear and circular polarizations. The parameters of the phase modulation are: $180 \mathrm{MHz}(260$ $\mathrm{MHz})$ and $300 \mathrm{MHz}(200 \mathrm{MHz})$ at $589 \mathrm{~nm}$; and $125 \mathrm{MHz}(225 \mathrm{MHz})$ at 569 $\mathrm{nm}$ (Beacon code).

In both cases, the circular polarization increases the return flux at $330 \mathrm{~nm}$ up to $40 \%$ for phase modulated lasers. It is also worth noting that the value of the spot diameter giving the maximum return flux is unchanged after polarization change. Again the best result in terms of flux and spot size is obtained for the modeless laser.

\subsection{Influence of the spectral width}

In this paragraph, we discuss the benefits of adjusting the spectrum of the modeless laser at $589 \mathrm{~nm}$ relatively to the $\mathrm{D}_{2}$ line. Up to now, we considered a Gaussian laser spectral line with a $3 \mathrm{GHz}$ FWHM to excite all velocity classes associated to the $3 \mathrm{~S}_{1 / 2} \rightarrow 3 \mathrm{P}_{3 / 2}$ transition. But a quick look shows that the overlap of a Gaussian function with the double-Gaussian shape of the $\mathrm{D}_{2}$ line is not optimal. Therefore we investigated the possibility of exciting only the $\mathrm{F}=2$ line of the $\mathrm{D}_{2}$ transition. (This configuration happens to be the most efficient for the phase modulation; see §3.1.) This line is a regular Dopplerbroadened Gaussian function with a $1 \mathrm{GHz}$ FWHM. We compare the return fluxes vs. spot size for the $3 \mathrm{GHz}$ and for the $1 \mathrm{GHz}$ spectral width of the first laser. Note that the second laser remains unchanged (modeless, $1 \mathrm{GHz}$ spectral width). The results are presented in fig. 18. 


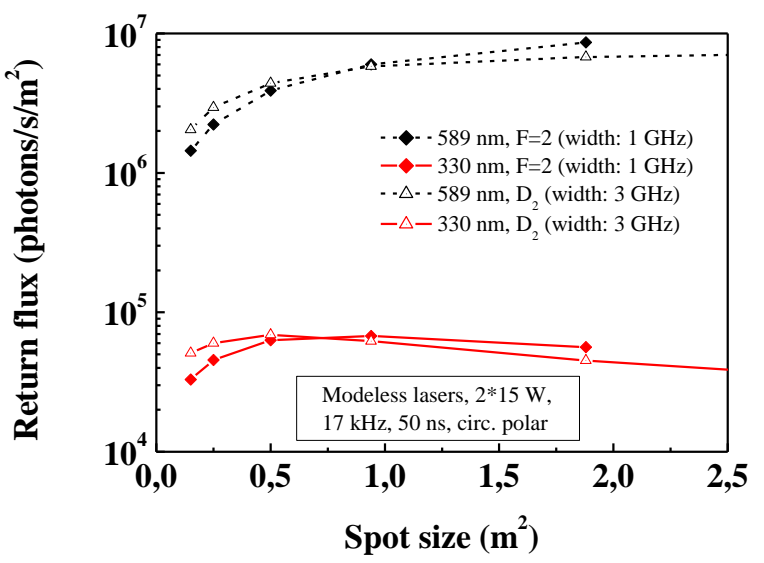

Fig. 18. Return flux at 589 and $330 \mathrm{~nm}$ with respect to the spot size. In one case (diamond), the modeless laser at $589 \mathrm{~nm}$ is centred on the $\mathrm{F}=2$ lobe of the $\mathrm{D}_{2}$ line $(\mathrm{FWHM}=1 \mathrm{GHz})$. In the second case (triangles), it covers the whole $\mathrm{D}_{2}$ line with a $3 \mathrm{GHz}$ FWHM. In both cases the second laser is modeless with a 1 $\mathrm{GHz}$ FWHM centred on the transition at $569 \mathrm{~nm}$.

Narrowing the spectral width of the first laser leads to the following behaviours. First, the return flux is decreased for small laser spot sizes (i.e. in the saturated regime). This is easily understandable since the energy is distributed over less velocity classes and the saturation is increased. On the side of large laser spots (i.e. linear regime), the return flux for both 589 $\mathrm{nm}$ and $330 \mathrm{~nm}$ is increased when narrowing the laser spectrum. This is mainly due to the better overlap between the laser and the absorption line. Note also that the maximum of the fluorescence at $330 \mathrm{~nm}$ remains unchanged, but its position is shifted by a factor of $\sim 2$ on the horizontal axis. In the frame of the polychromatic LGS where the flux has to be maximized for a minimal spot surface, the saturation is the main limiting factor. Even if the spectrum is not ideally matched, the $3 \mathrm{GHz}$ width laser is still preferable.

\subsection{Influence of the pulse duration}

The influence of the pulse duration on the return flux has also been checked. For identical laser powers and repetition rates, we compare two different pulse durations: the first one is $50 \mathrm{~ns}$, the second one $80 \mathrm{~ns}$. On one side, it is desirable to increase the pulse duration to reduce the saturation; on the other side, $80 \mathrm{~ns}$ appears as an intrinsic limit to the current dye amplifier systems. Both pulse durations have been compared, all parameters being kept constant (energy per pulse, spot size, linear polarization, modeless laser). $80 \mathrm{~ns}$ long laser pulses lead to a slight increase of the fluorescence: the relative difference between the return fluxes reaches $20 \%$ in the strong saturation limit.

\subsection{Influence of the delay between the laser pulses}

The delay between the laser pulses can also be varied in the Beacon and REM code. We studied the case where both lasers pulses are $50 \mathrm{~ns}$ long and we delayed the second laser pulse by steps of $5 \mathrm{~ns}$, from a negative delay of $5 \mathrm{~ns}$, to positive ones, up to $20 \mathrm{~ns}$. The maximum relative difference in the return fluxes at $330 \mathrm{~nm}$ does not exceed $5 \%$, which shows that the two photon excitation process with tens of ns laser pulses is very robust with respect to the temporal mismatch of the laser pulses.

\subsection{Influence of the repartition of the laser intensity}

Similarly we also checked the influence of the repartition of the laser intensity. Since the transition strengths of the $589 \mathrm{~nm}$ and $569 \mathrm{~nm}$ are quite different -a factor of ten in the absorption cross section-, we investigated the possibility of increasing the return flux for a given total laser power, by changing the relative intensities of both lasers. We compare three situations. In the first one, both lasers have the same average power $(15 \mathrm{~W}$ each). In the second one, the first laser (at $589 \mathrm{~nm}$ ) has an average power of $20 \mathrm{~W}$, the second one of $10 \mathrm{~W}$. The third case is the opposite situation, with the first laser delivering $10 \mathrm{~W}$ and the second one $20 \mathrm{~W}$. Situations 2 and 3 give extremely close results, differing by less than $2 \%$. But in both cases, the return flux at $330 \mathrm{~nm}$ remains lower than in the first case; the relative differences remaining below $10 \%$. This suggests that the laser power has to be equally distributed between both lasers.

\section{Conclusion}

The first point we would like to underline is the good agreement between the Bloch equation treatment of the PLGS (Beacon) and the simplified rate equation model (REM) developed in the frame of [6]. The qualitative agreement is particularly obvious; the relative behaviours are in very good agreement. From a quantitative point of view, the relative difference is about $50 \%$. This conclusion is of importance since the calculation time ( 24 hours) is the main limitation to the use of the Beacon code available online. The calculation time of the REM is about 1 minute. In specific cases the return flux budget of the PLGS requires the complete Bloch equation model (circular polarization for instance) but the REM is sufficient in numerous cases. The REM model will be published and available in a forthcoming paper.

Concerning the possible implementation of the PLGS by the two-photon resonant excitation scheme, an important parameter needs to be taken into account: the size of the laser spot at the mesosphere. The key point to consider is not the maximum return flux, but the ratio of the return flux divided by the surface of the fluorescence spot. Indeed the uncertainty on the tip-tilt determination is linked to both the photon noise and the size of the fluorescence spot. It is beyond the scope of this paper to discuss this in detail, and further studies would be required to link the size of the fluorescence spot to the size of the laser beams at the mesosphere, after propagation through the turbulent layers of the atmosphere. However one can see from the shape of the return flux at $330 \mathrm{~nm}$ with respect to the laser spot size that maximizing the return flux usually implies 
increasing the laser spot size. Both parameters have to be considered together.

Numerous simulations have been run to determine the maximal return flux for a given (reasonable) laser power. The results of these studies show that the values published previously have been overestimated [5]. The results given in the latter reference are plotted in fig. 13. For clarity, we also recall in table 2 the different flux expectations for the three cases considered in [5]. Instead of $2.210^{6}$ photons $/ \mathrm{s} / \mathrm{m}^{2}$ (resp. $2.210^{5}$ photons $/ \mathrm{s} / \mathrm{m}^{2}$ ), for a spot surface of $0.049 \mathrm{~m}^{2}$ [5], Beacon leads to a maximal flux of $4.310^{4}$ photons $/ \mathrm{s} / \mathrm{m}^{2}$ (resp. $3.210^{4}$ photons $/ \mathrm{s} / \mathrm{m}^{2}$ ) for $80 \mathrm{~ns}$, $30 \mathrm{kHz}, 2 * 18 \mathrm{~W}$, modeless (resp. phase-modulated) lasers, tophat profile, circular polarization (ELPOA 3 and 2). For the case ELPOA 1, [5] gives a return flux equal to $510^{4}$ photons $/ \mathrm{s} / \mathrm{m}^{2}$ (for $50 \mathrm{~ns}, 17 \mathrm{kHz}, 2 * 15 \mathrm{~W}$, phase modulation, linear polarization), to be compared to the flux given by Beacon: 1.4 $10^{4}$ photons $/ \mathrm{s} / \mathrm{m}^{2}$. (Note that concerning phase modulation, the optimal return fluxes are obtained with very strong PM intensities, which imposes to apply to the EO crystals voltages exceeding the nominal characteristics.) Recall that a circular polarization would lead to a slight increase of the return flux in this latter case. (In both cases, considering a gaussian laser spot at the mesosphere would lead to an improvement of the flux by a factor of about 2, but the effective size of the fluorescence spot would also increase.) For a spot ten times larger $\left(0.5 \mathrm{~m}^{2}\right)$, and for a $2 * 18 \mathrm{~W}$ laser, in any case the return flux at $330 \mathrm{~nm}$ should not exceed $7.10^{4}$ photons $/ \mathrm{s} / \mathrm{m}^{2}$. Finally, due to several technical problems (spatial, spectral, time overlap ...), the experimental result should be less than this theoretical prediction.

\begin{tabular}{|l|c|l|l|l|}
\hline & \multirow{2}{*}{$\begin{array}{c}\text { Values reported } \\
\text { in [5]: }\end{array}$} & \multicolumn{3}{|c|}{ Beacon results: } \\
\cline { 3 - 5 } & & PM (a) & PM (b) & PM (c) \\
\hline ELPOA 1 & $510^{4}$ & $6.610^{3}$ & $8.210^{3}$ & $1.410^{4}$ \\
\hline ELPOA 2 & $2.210^{5}$ & $1.810^{4}$ & $2.010^{4}$ & $3.210^{4}$ \\
\hline ELPOA 3 & $2.210^{6}$ & & & $4.310^{4}$ \\
\hline
\end{tabular}

Table 2. Comparison of the return flux at $330 \mathrm{~nm}$ given in [5], with the Beacon results. All fluxes are given in photons $/ \mathrm{s} / \mathrm{m}^{2}$. The parameters of ELPOA-1 to 3 are given in the text. Three different phase modulations have been tested, the first one corresponds to the values of the PM frequencies used in PASS-2 [18]. PM (a): laser 1: $180 \mathrm{MHz}(260 \mathrm{MHz})$ and $300 \mathrm{MHz}(200 \mathrm{MHz})$, laser 2: 125 MHz (250 MHz). PM (b): $180 \mathrm{MHz}(260 \mathrm{MHz})$ and $300 \mathrm{MHz}(200 \mathrm{MHz})$ for both lasers. PM (c): $150 \mathrm{MHz}(1300 \mathrm{MHz})$ and $110 \mathrm{MHz}(500 \mathrm{MHz})$ for both lasers [27]. See $\$ 3$ for notations.

Schöck and colleagues have demonstrated that a return flux of $510^{4}$ photons $/ \mathrm{s} / \mathrm{m}^{2}$ at $330 \mathrm{~nm}$ from a $0.049 \mathrm{~m}^{2}$ laser spot enables to obtain a Strehl ratio in the $\mathrm{V}$-band equal to $1 \%$ (case ELPOA 1), and to $5 \%$ with a return flux of $2.210^{5}$ photons $/ \mathrm{s} / \mathrm{m}^{2}$ (ELPOA 2). Recall that these calculations suppose a $1.52 \mathrm{~m}$ diameter reception telescope with an AO system, and a diffraction-limited laser beam at the mesosphere $\left(0.049 \mathrm{~m}^{2}\right.$ spot size). From $\$ 2.3$ and fig. 13 , we can deduce the laser power and repetition rate necessary to provide the requested fluxes. We consider a laser system delivering $80 \mathrm{~ns}$ pulses, with phase modulation and circular polarization. For the case ELPOA-1, we deduce that $2 * 25 \mathrm{~W}$ at the ground with a repetition rate of $10 * 30=300 \mathrm{kHz}$ should provide the requested flux $\left(5.10^{4}\right.$ photons $\left./ \mathrm{s} / \mathrm{m}^{2}\right)$ with a spot size of $0.05 \mathrm{~m}^{2}$. For the case ELPOA-2, a theoretical return flux equal to $2.210^{5}$ photons $/ \mathrm{s} / \mathrm{m}^{2}$ at $330 \mathrm{~nm}$ with a $0.05 \mathrm{~m}^{2}$ laser spot can be obtained by $2 * 4 * 25=2 * 100 \mathrm{~W}$ total laser power and a $4 * 10 * 30=1200 \mathrm{kHz}$ phase-modulated laser system, which seems quite challenging. A similar flux could be obtained by two modeless lasers with slightly more favourable parameters: $2 * 3 * 25=2 * 75 \mathrm{~W}$ total power and $3 * 4 * 30=360 \mathrm{kHz}$ repetition rate. But in any case, high repetition rates are a problem for dye systems.

Finally, photometry experiments are in progress in our laboratory. Their results will be published shortly, along with the corresponding numerical results given by both the REM and the Beacon code. They will be compared to the experimental results of PLGS obtained in the past years [23,27,28].

\section{Acknowledgements}

We would like to thank Alain Petit and Véronique Bellanger, from the Commissariat à l'Energie Atomique for useful discussions and remarks. This work was supported by the CNRS (SPM, MRCT).

\section{References}

[1] F. Rodier (ed.), Adaptive Optics in Astronomy (Cambridge Univ. Press, 1999).

[2] R. Foy and A. Labeyrie, "Feasibility of adaptive telescope with laser probe", Astron. Astrophys. 152, L29-L31 (1985). L. Thompson and C. Gardner, "Experiments on laser Guide Stars at Mauna Kea Observatory for Adaptive Optics in Astronomy", Nature 328, 229-235 (1987).

[3] F. Rigaut, E. Gendron, "Laser guide star in adaptive optics: the tilt determination problem", Astron. Astrophys. 261, 677 (1992).

[4] R. Foy, A. Migus, F. Biraben, G. Grynberg, P. R. McCullough, and M. Tallon, "The polychromatic artificial sodium star: a new concept for correcting the atmospheric tilt," Astron. Astrophys. Suppl. Ser. 111, 569-578 (1995).

[5] M. Schöck, R. Foy, M. Tallon, L. Noethe and J.-P. Pique, "Performance analysis of polychromatic laser guide stars used for wavefront tilt sensing," Mon. Not. R. Astron. Soc. 337, 910-920 (2002).

[6] J. P. Pique, I. Moldovan, V. Fesquet, "Concept for polychromatic laser guide stars: one-photon excitation of $4 \mathrm{P}_{3 / 2}$ level of a sodium atom", J. Opt. Soc. Am. A, 23,2817-2827 (2006).

[7] G. Froc, E. Rosencher, B. Attal-Trétout, V. Michau, "Photon return analysis of a polychromatic laser guide star," Opt. Comm. 178, 405-409 (2000). 
[8] V. Bellanger, A. Courcelle, A. Petit, «A program to compute the two-step excitation of mesospheric sodium atoms for the Polychromatic Laser Guide Star Project," Comp. Phys. Comm. 162, 143-150 (2004).

[9] N. Ageorges, N. Hubin, "Atmospheric sodium monitor for Laser Guide Star Adaptive Optics," Astron. Astrophys. Suppl. Ser. , 533-540 (2000).

[10] L. Michaille, A. D. Cañas, J. C. Dainty, J. Maxwell, T. Gregory, J. C. Quartel, F. C. Reawell, R. W. Wilson and N. J. Wooder, "A laser Beacon for monitoring the mesospheric sodium layer at La Palma," Mon. Not. R. Astron. Soc. 318, 139-144 (2000).

[11] J. Biegert and J. C. Diels, "Feasibility study to create a polychromatic guidestar in atomic sodium," Phys. Rev. A 67, 043403 (2003).

[12] B. Cagnac, J. P. Faroux, Lasers (CNRS Editions, 2002), pp. 495-498.

[13] P. W. Milonni, J. H. Eberly, Lasers (Wiley, 1988), pp. 224-227.

[14] R. C. Hilborn, "Einstein coefficients, cross sections, $f$ values, dipole moments and all that," Am. J. Phys. 50, 982-986 (1982), R. C. Hilborn, "Erratum: Einstein coefficients, cross sections, $f$ values, dipole moments and all that," Am. J. Phys. 51, 471 (1983).

[15] P. L. Wizinovitch, D. Le Mignant, A. H. Bouchez, R. D. Campbell, J. C. Y. Chin, A. R. Contos, M. A. Van Dam, S. K. Hartman, E. M. Johansson, R. E. Lafon, H. Lewis, P. J. Stomski and D. M. Summers, "The W. M. Keck observatory laser guide star adaptive optics system: overview," Pub. Astron. Soc. Pac. 118, 297-309 (2006).

[16] J. Drummond, J. Telle, C. Denman, P. Hillman and A. Tuffli, "Photometry of a sodium laser guide star at the Starfire Optical Range," Pub. Astron. Soc. Pac. 116, 278-289 (2004). M. Boccas, F. Rigaut, M. Bec, B. Irarrazaval, E. James, A. Ebbers, C. d'Orgeville, K. Grace, G. Arriagada, S. Karewicz, M. Sheehan, J. White, S. Chan, "Laser Guide Star upgrade of Altair at Gemini North," in Advances in Adaptive Optics II, B. L. Ellerbroek and D. Bonaccini Calia Eds., Proc. SPIE 6272 (2006).

[17] J. R. Morris, "Efficient excitation of a mesospheric sodium laser guide star by intermediate-duration pulses," J. Opt. Soc. Am. A, 11, 832-844 (1994).
[18] V. Bellanger, University of Paris VI, PhD thesis (2002, unpublished).

[19] J. P. Pique and S. Farinotti, "Efficient modeless laser for a mesospheric sodium laser guide star," J. Opt. Soc. Am. B, 20, 2093-2102 (2003).

[20] F. V. Kowalski, P. D. Halle and S. J. Shattil, "Broadband continuous-wave laser," Opt. Lett. 13, 622-624 (1988).

[21] S. Balle, I. C. M. Littler, K. Bergmann, F. Kowalski, "Frequency shifted feedback dye laser operating at a small shift frequency," Opt. Comm. 102, 166-174 (1993).

[22] L. P. Yatsenko, B. W. Shore, K. Bergmann, "Theory of a frequency-shifted feedback laser," Opt. Comm. 236, 183-202 (2004).

[23] M. Schöck, R. Foy, J. P. Pique, P. Chevrou, N. Ageorges, A. Petit, V. Bellanger, H. Fews, F. C. Foy, C. Högemann, M. Laubscher, O. Peillet, P. Segonds, M. Tallon, J. M. Weulersse, "PASS-2: Quantitative photometric measurements of the polychromatic laser guide star," in Adaptive Optics Technology, P. L. Wizinovitch Ed., Proc. SPIE 4007 (2000).

[24] H. Guillet de Chatellus and J.P. Pique, "Bilan du flux retourné par les étoiles laser polychromatiques", presented at the $10^{\text {th }}$ Conference on lasers and quantum optics (COLOQ 10), Grenoble, France, 2-5 July 2007.

[25] A. E. Siegman, Lasers (University Science Books, 1986), pp. 663-667.

[26] R. Foy, CRAL/Observatoire de Lyon, 9 avenue Charles André, 69561 Saint Genis-Laval, France (personal communication, 2006).

[27] R. Foy, M. Tallon, I. Tallon-Bosc, E. Thiébaut, J. Vaillant, F. C. Foy, D. Robert, H. Friedman, F. Biraben, G. Grynberg, J. P . Gex, A. Mens, A. Migus, J. M. Weulersse, D. J. Butler, "Photometric observations of a polychromatic laser guide star”, J. Opt. Soc. Am. A 17, 2236-2242 (2000).

[28] R. Foy, J. P. Pique, V. Bellanger, P. Chevrou, A. Petit, C. Högemann, L. Noethe, M. Schöck, J. Girard, M. Tallon, E. Thiébaut, J. Vaillant, F. C. Foy and M. Van Dam, "Feasibility study of the polychromatic laser guide star," in Adaptive Optical System Technology II, P. L. Wizinovitch, D. Bonaccini Eds., Proc. SPIE 4839 (2003). 